(Aus dem Hamburge:: Institut für Krebsforschung.)

\title{
Zur Energetik der Bildung maligner Tumoren.
}

\author{
Von \\ Dr. R. Bierich.
}

\section{Einleitung.}

Die verschiedenen organischen Bildungen, an denen hier das Problem des malignen Wachstums untersucht wurde, haben ihr gemeinsames Kriterium darin, da B es kolloidale Systeme sind.

Um das Wesen der ihren formalen Bildungen offenbar zugrunde liegenden Gesetzmäßigkeiten zu erkennen, wurde versucht, diese auf die physikalisch-chemischen Eigenschaften der Struktur zurückzuführen.

Aus dem strukturellen Aufbau mußte sich dann voraussichtlich eine Vorstellung von der Art und der Wirkungsweise der ihn bestimmenden Energien gewinnen lassen.

Die Fragestellung konnte also dahin formuliert werden, ob sich die Entstehung des malignen Wachstums auf den Ausgleich der in den beteiligten Systemen gegebenen Energien zurückführen läßt.

Vom gleichen Gesichtspunkt aus wurden im Verlauf der Untersuchung ebenso die allgemeinen biologischen Probleme der Geschwulstlehre - das Wachstum der Tumoren, die Allgemeinschädigung des Organismus - zu erklären gesucht. - Wenn hier von der Annahme ausgegangen wird, daß es sich bei der pathologischen Neubildung um das Reaktionsprodukt verschiedenwertiger Energiesysteme handelt, von denen das eine durch das Eingreifen des andern mittelbar zur Ausbildung neuer Funktionen veranlaßt wird, so mußte als Basis eine Vorstellung darüber gewonnen werden, in welchen speziellen Formationen des Tumors, resp. seines Bodens, diese Energien vorliegen und welcher Art sie sind. Diese Energien konnten, retrospektiv in latenten entwicklungsmechanischen Potenzen, oder in den bereits differenzierten Strukturen des Organismus gegeben sein. Damit war die alte Kontroverse erneut zu entscheiden, ob es sich bei der Entstehung maligner Tumoren um das Inkrafttreten entwicklungsmechanischer latenter Energien oder um die Bildung neuer Energieformen aus bereits funktionierenden handelte, also um eine unter bestimmten Bedingungen an entwicklungsmechanisch nicht prädisponierter Stelle eintretende Umbildung vorhandener Energiesysteme. Es wurde demnach der Versuch gemacht, 1. die entwicklungsmechanischen Potenzen, 2. die physikalisch-chemischen Potenzen der 
reagierenden Systeme, 3. das Wesen der „Reizfaktoren“ und die wechselseitige Reaktion von "Reizfaktor" und Gewebe näher zu definieren. Die Strukturänderungen, die bei der Tumorbildung am Protoplasma der beteiligten Zellsysteme zu beobachten sind, erklären sich am besten als Resultate eines physikalisch-chemischen Ausgleichs zwischen energetisch verschiedenwertigen Kolloidsystemen. Aus der Strukturänderung - d. h. dem formalen Eindruck der Strukturumlagerung - muß auf eine gleichzeitige Funktionsänderung des Systems geschlossen werden, da die Funktionen eines kolloidalen Systems direkte Folgen seiner energetischen Struktur sind. Bestimmte Angaben über die quantitativen und qualitativen Eigenschaften des neugebildeten Produkts lassen sich zur Zeit nicht machen, erscheinen auch - im Vergleich zum heuristischen Wert der gewonnenen prinzipiellen Anschauung, von sekundärer Bedeutung.

\section{Die entwiekJungsmechanischen Potenzen der reagierenden Systeme.}

Da das Bindegewebe, neben seiner mechanischen Funktion als Stützgewebe, durch Einlagerung der Ernährungs- und der nervösen Regulierungsbahnen der Organe, in denen Schutz- und Abwehrstoffe des Organismus gebildet werden, ein allem Anschein nach den übrigen Organen gleichwertiges, selbständiges Organ ist, lag es nahe, vor Untersuchung der epithelialen Gewebe, an deren Aufbau es durchweg beteiligt ist, seine Reaktion auf energetische Schädigungen zu untersuchen. Vor allem mußte Stellung dazu genommen werden, ob seine aktive oder passive Reaktion auf in ihm latente entwicklungsmechanische Potenzen zurückführbar ist.

Als Basis diente das Studium des embryonalen Bindegewebes, und die hier beobachteten Bildungen wurden dann, in bezug auf ihre Differenzierungsmöglichkeit, an den gleichen Formationen späterer Entwicklungsstadien - bei jugendlichen und erwachsenen Individuen weiter verfolgt. Als Objekte wurden von Kaltblütern: Frosch- und Fischembryonen, von Warmblütern: Mäuse und Ratten untersucht. Das Gewebe wurde lebenswarm entnommen und sowohl in Zupfpräparaten ungefärbt - in physiol. NaCl-Lösung, - nach Säure- oder Alkalizusatz - oder nach voraufgegangener Vitalfärbung untersucht. In einer zweiten Serie wurden die Gewebsstrukturen nach Bichromat- oder Carnoyfixierung in Schnitten studiert. Für Protoplasmafärbungen wurde Methylgrün-Pyronin, Azur-Eosin, Heidenhains Hämatoxylin, Hämatoxylin-Eosin - für Bindegewebsfärbungen vor allem AchucarroRancke benutzt. Die hierbei gewonnenen Anschauungen von dem Bildungsmodus des Bindegewebes decken sich im wesentlichen mit den neueren Anschauungen Heide nhains ${ }^{11}$ ), v. Szil ys ${ }^{38}$ ), Studnickas ${ }^{37}$ ), Rhodes ${ }^{33}$ ), Ranckes ${ }^{30}$ ), Huecks ${ }^{17}$ ), Meves ${ }^{24}$ ) u.a. Mit diesen haben 
wir uns die ganze Protoplasmamasse des Embryos in der ersten Anlage als schwammartige, syncytiale Bildung vorzustellen, wobei die Keimblätter ein zusammenhängendes Netz mit eingelagerten Kernen bilden. Dieses Netz verbindet nicht nur die Kerne in flächenhafter Anordnung zu den Keimblättern, sondern verbindet ebenso die Keimblätter der Tiefe nach. Dieses zwischengelagerte ,epitheliale“ Bindegewebe [Held $\left.\left.{ }^{12}\right)\right]$ erscheint anfangs als strukturloses Maschenwerk, in das später aus den Keimblättern Kerne einwandern, die sich besonders in den Kreuzungspunkten der Netzfasern finden. Damit erscheint das Bindegewebe in seiner ersten Anlage organisiert. Die Grundsubstanz in den Hohlräumen der ganzen schwammartigen Anlage ist aufzufassen als sekundäre Verflüssigung des bindegewebigen Netzes, oder sie entsteht durch periodische oder kontinuierliche Abscheidung des Protoplasmas. Eine solche unitarische Auffassung der gesamten Anlage im Embryo gibt dann dem Bindegewebe entwicklungsmechanisch den Charakter einer epithelialen Bildung [Held $\left.\left.{ }^{12}\right)\right]$ und damit nicht nur die Funktionsanlage eines selbständigen Organs, sondern auch die übrigen Bildungsmöglichkeiten eines solchen. Es wird weiter zu bestimmen sein: 1. wie weit diese Bildungsmöglichkeiten gehen können, und 2., ob sie in dem ganzen Gewebe verstreut liegen oder auf bestimmte formal erkennbare Bezirke beschränkt sind. Verfolgt man die Entwicklung des anfänglich zarten Maschenwerks mit den in seinen Knotenpunkten eingelagerten Kernen, so machen sich zuerst Veränderungen an den Fasern selbst bemerkbar. An ihrer Grenzfläche gegenüber der Grundsubstanz treten Verdichtungen auf und im Innern erscheinen stärker lichtbrechende faserartige Bildungen, die beide, strukturlos und kernfrei, in späteren Stadien ihre Differenziertheit durch spezielle Färbungen als kollagene und elastische Fasern und Membranen nachweisen lassen: Zur Erklärung dieser Bildung müssen wir zum Teil ṫberlegungen des folgenden Absatzes vorwegnehmen.

In dem Komplex - Fasernetz und Grundsubstanz - liegt eine protoplasmatische, also kolloidale Bildung vor. Wir haben diese als ein wenigstens zweiphasisches kolloidales System aufzufassen, für das die bekannten physikalisch-chemischen Gesetze der Grenzflächen gelten. An der Berührungsfläche zweier verschieden disperser Phasen haben wir Energien vom Typus der Adsorptionspotentiale oder der elektrischen Doppelschicht anzunehmen, und diese Energien erklären ohne weiteres die Bildung der Verdichtungserscheinungen an der Grenze zwischen Faser und Grundsubstanz. Die Bildung kollagener und elastischer Fasern in den Netzbalken muß dagegen rein mechanisch erklärt werden - sie entstehen durch Kondensation überall da, wo die Struktur des Gewebes in bestimmten Richtungen stärker belastet wird. Aus dieser Auffassung läßt sich vor allem ein Verständnis für die Lebensfunktionen des 
ganzen Systems gewinnen, denn überall an der Berührungsfläche differenter Phasen haben wir Energien vom Typus der $\mathrm{Helmholtzschen}$ Doppelschicht anzunehmen.

Für unsere Aufgabe handelt es sich, abgesehen von diesen später zu besprechenden sekundären Bildungserscheinungen, um den Nachweis von entwicklungsmechanisch garantierten Bildungsmöglichkeiten. Von früheren Autoren wurde angenommen, daß das Bindegewebe rein passive Stützfunktion hat und an ihm keinerlei Fortbildungen, sondern nur Rückbildungen möglich sind.

Nun sprechen ja schon die eben entwickelten Vorstellungen von der Differenzierungspotenz gegen solch eine Auffassung, abgesehen davon, daß überhaupt nicht anzunehmen ist, daß irgend ein Gewebe im Körper kein eigenes Leben besitzt.

Es erscheint hier unnötig, für die Entstehung des fibrillären Bindegewebes, der elastischen Fasern, andere als die erwähnten, energetischen Momente anzunehmen, besonders da in dem Protoplasma selbst eine Kondensationstendenz vorliegt; es entsteht spontan und ist $\mathrm{zu}$ weiteren Kondensationen durch seine Struktur veranlagt [E. Fischer $\left.\left.{ }^{9}\right)\right]$. Während solche Kondensationsvorgänge für unsere Frage nach dem Ausgangspunkt entwickelungsmechanischer Potenzen von sekundärer Bedeutung sind, kommt den nunmehr zu besprechenden Anlagen des Ernährungssystems und des umgebildeten Stützgewebes eine wesentlich höhere Wichtigkeit für die Reaktion gegenüber einem „Reizfaktor"“ zu; dies sind Lymph- und Blutgefäße, die glatten Muskelfasern, Knorpel, Knochen und die drüsenartigen Anlagen. Die Entstehung dieser

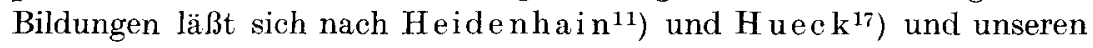
Untersuchungen folgendermaßen ableiten: Aus dem primären schwammartigen Gerüst wandern in die Poren Zellen ab, um die sich die Gerüstsubstanz zu membranartigen syncytialen oder netzartigen Bildungen verdichtet. Die Kerne nehmen dabei allmählich die für Muskelzellen typische stäbchenförmige Gestalt an.

Ähnlich liegen dann wohl auch die Verhältnisse für die Bildung von Knorpel und Knochen. Das primäre dürfte dabei die von Hartmann ${ }^{10}$ ) und v. Korff ${ }^{19}$ ) beschriebene mechanische Umordnung der fibrillären Substanz des Bindegewebes sein. Diese wird nach statischen Gesetzen bogenförmig oder spiralig umgelagert, die Poren werden dann zu geschlossenen Höhlen und in diese neugruppierten Bildungen wandern aus dem Schwammgerüst Kerne ein, die die erste Anlage von Chondro- oder Osteoblasten bilden. Die Grundsubstanz wird durch Aufnahme spezieller chemischer Verbindungen zu einem homogenen, chondrogenen oder osteoiden Gewebe.

Bei der postembryonalen Bl utbild ung kommen graduelle Änderungen dieses Prozesses insofern in Betracht, als die aus dem Schwamm. 
gerüst auswandernden Zellen sich völlig loslösen und dann frei in den Poren liegen (Mollie $\left.{ }^{24}\right)$ ]. $-\mathrm{Hueck}^{17}$ ) nimmt z. B. für die Lymphocyten an, daß sie hierbei aus dem retikulären Apparat der Gewebe (den Keimzentren) hervorgehen. Die, für die Entzündungsvorgänge von Marchand ${ }^{21}$ ) und Herzog ${ }^{14}$ ) angeführten Beobachtungen decken sich mit diesen und unseren - bei den Teercarcinomen gemachten Beobachtungen. Hueck weist dabei ausdrücklich darauf hin, daß die Blutzellenbildung nur in solchen wenig differenzierten Zentren anzunehmen ist, die einen dem fötalen ähnlichen Charakter bewahrt haben, also vorzugsweise im retikulären Bindegewebe, sowohl in seiner allgemeinen Anordnung im Gewebe wie in den speziellen in der Adventitia der Capillaren [Herzogit), Marchand ${ }^{22}$ )]. - Die Frage der Blut- und Lymphgefäßbildung im embryonalen Organismus ist noch offen; nach Meinung der neueren Autoren [Rückerti1), Mollier ${ }^{24}$ \}, $\mathrm{Hueck}^{17}$ )] entstehen die Blutgefäße sowohl aus der Peripherie der ,Area opaca“, und die Lymphgefäße als Sprossungen der Venen, - als auch autochthon im Bindegewebe, wo ,durch Resorption von $Z$ wischenwänden bestimmte Reihen von Poren auf das gleiche Kaliber gebracht werden und so . . cylindrische Rohre bilden“. Dabei nimmt Hueck an, daß das ,die Poren begrenzende Schwammgewebe zur Gefäßwand verdichtet wird, während die das Lumen begrenzenden protoplasmatischen Anteile des Schwam. mes eng zusammengeschoben werden und das geschlossene Endothelrohr bilden". Im erwachsenen Organismus werden solche Bildungen generell nicht auftreten, sondern sich nur da beobachten lassen, wo junges, undifferenziertes Gewebe vorliegt oder sich bildet. Bedingungen hierfür sind im Granulationsgewebe, sowohl um Fremdkörper [Her$\left.\left.\left.\operatorname{zog}^{14}\right), B \operatorname{Brst}^{3}\right)\right]$, wie in Granulations- und echten Geschwülsten [H uec $\left.\left.\mathrm{k}^{17}\right)\right]$, unsere später anzuführenden Befunde, gegeben.

Die hier geschilderten Bildungsmöglichkeiten waren in erster Linie qualitative, die Bildung von Muskel, Knorpel, Knochen, Gefäßen und Blutzellen war dabei beschränkt auf das embryonale Stadium und im erwachsenen Organismus auf solche Zustände, wo an umschriebener Stelle wieder niedrig differenziertes, retikuläres Bindegewebe auftrat. Die quantitative Bildungsfähigkeit erschien dabei für das Bindegewebe beschränkt.

Wenn eine Reaktion von seiten des Bindegewebes eintritt, wie z. B. im Granulationsgewebe, so wird diese erst nach Bildung eines möglichst undifferenzierten Gewebes möglich; das hochdifferenzierte Bindegewebe des erwachsenen Organismus ist aus sich heraus nur imstande, dem Endstadium der Differenzierung zuschreitende Kondensationsprodukte zu bilden, die also das labile Gleichgewicht des undifferenzierten Energiesystems immer mehr verlieren und stabileren, d. h. energieärmeren Zuständen entgegengehen. 
Die entwicklungsmechanischen Bildungsmöglichkeiten sind also nach diesem indirekten Maßstab für das Bindegewebe des erwachsenen Organismus gering. Aber auch im embryonalen Zustand oder unter Bedingungen, wo das Bindegewebe wieder sich dessen qualitativen Bildungsmöglichkeiten nähert, sind nur beschränkte qualitative Bildungen möglich, die über die Grenze der in dem embryonalen Gewebe gegebenen, ontogenetisch begrenzten Bildungen nicht hinausgehen. Da wir andererseits eine Reihe den epithelialen Neubildungen in lokaler Wachstumsenergie, Aggressivität und Metastasierfähigkeit ebenbürtige maligne und benigne Geschwulstbildungen der Bindegewebegruppe kennen, fragt es sich, ob für diese, abgesehen von den entwicklungsmechanisch ableitbaren Energien, nicht irgendwelche andere formale Energiezentren nachweisbar sind.

Die theoretischen Vorstellungen von den Qualitäten des Ausgangsmaterials des pathologischen Wachstums gingen im wesentlichen von einem relativ in sich abgeschlossenen System aus, - den entwicklungsmechanischen embryonalen oder postembryonalen Fehl- oder Miß. bildungen. - Es ist unbestreitbar, da $\beta$ in diesen ,,isolierten" Formationen das Ausgangsmaterial pathologischer. Wachstumsprozesse gegeben sein kann und oft gegeben ist, aber in ihnen ist doch nur eine Materialart gegeben. Abgesehen davon, daß solche Komplexe sich während des ganzen Lebens völlig passiv müssen verhalten können, denn sonst müßten an jedem menschlichen Individuum zumindest einige pathologische Geschwulstbildungen zu beobachten sein, - geht eine solche Fragestellung auf das formale Ausgangsmaterial, während das Wesen seiner Wachstumsfähigkeit zur Diskussion stehen sollte. Wenn eine solche, nach den bisherigen Ausführungen in den allgemeinen entwicklungsmechanischen Potenzen der embryonalen oder postembryonalen Zellen und Gewebe nur in beschränktem Grade nachgewiesen werden konnte, war ja wohl die Möglichkeit nicht auszuschlieBen, sie in speziellen Wachstumszentren aufzudecken.

Diesbezüglichen Versuch machte Schaper ${ }^{32}$ ) durch den Nachweis der "Indifferenzzonen". Es lassen sich beim Embryo ausgedehnte, in einfachen epithelialen Verbänden meist diffuse Verteilungen von Karyokinesen nachweisen, die mit der Differenzierung der Organe abnehmen und speziell im postembryonalen Zustande gesetzmäBig auf bestimmte Abschnitte der einzelnen Organe beschränkt sind. Solche Zellen garantieren die Wachstumsbereitschaft des betreffenden Organs und regulieren nach Bedarf den Nachschub der im Organbetrieb oder bei der physiologischen Regeneration notwendigen spezifischen Zellen. Sie sind demnach als Orte erhöhter Wachstumsenergie, als relativ undifferenzierter Teil, zur Bildung bestimmter Zellen befähigt. In ihnen wäre also ein System gegeben, von dem nach Bedarf - d. h. dem- 
nach periodisch - Wachstum in bestimmter Richtung und von bestimmter Qualität erfolgen kann. Ihre Bildungsmöglichkeiten mögen zwar im embryonalen Organismus omnipotent sein, aber mit fortschreitender Differenzierung und Übernahme spezieller Funktionen geht diese omnipotente Bildungsmöglichkeit und schließlich auch, vorübergehend oder dauernd, die Fähigkeit der Proliferation verloren [Schaper und Cohe $\left.{ }^{35}\right)$ ]. Eine allgemeine Wachstumsfähigkeit bleibt dabei nur in einfach organisierten Geweben, wie den einschichtigen Drüsengeweben und vielleicht einigen Bindegewebsarten, erhalten. Bei den anderen Geweben ist die Verteilung wachstumsfähiger Zellen (nach Schaper und Cohen) entweder diffus - wie im Bindegewebe und einigen einfach konstruierten Drüsen -, oder mehr auf die Randpartien der speziellen Gewebsanlage lokalisiert, wie beim geschichteten Epithel, dem Knorpel und Knochen (appositionelles Wachstum) oder auf bestimmte Gebiete beschränkt, wie in den Epiphysenlinien.

Bei epithelialen Organen, die nach ihrer komplizierten Form und der Spezialisierung ihrer Zellelemente hochwertige spezifische Funktionen besitzen, mußte eine Trennung des spezifisch funktionierenden Teils von dem neues Ersatzmaterial liefernden Teil angenommen werden, wodurch eine ungestörte Tätigkeit beider Komponenten erst garantiert war.

Diese Verhältnisse finden sich bei dem einschichtigen Epithel der serösen Höhlen, wo scheinbar sämtliche Zellen als proliferationsfähig anzunehmen sind, und andererseits beim geschichteten Epithel, wo die Wachstumsschicht an der Grenze zum Corium zu unterst liegt. Hier, wie bei allen anderen komplizierten Organen mit differenzierter Zelltätigkeit, sind die wachstumsfähigen Elemente gesetzmäßig so angeordnet, daß sie im indifferenten Abschnitt liegen, während der differenzierte keine Karyokinesen aufweist. Nimmt bei fortschreitender Entwicklung der anfangs breite indifferente Abschnitt ab, so bleibt immer eine schmale Indifferenzzone bestehen, die dann nach beiden Seiten den Nachschub garantiert. Die Indifferenzzonen einschichtiger Drüsenanlagen (Lieberkühnsche Krypten) zeigen $\mathrm{z}$. B. die Indifferenzzone immer auf das unterste Drittel der Krypte lokalisiert.

Dieselben Bedingungen, die für die physiologische Regeneration gelten, liegen nach Schaper für die pathologische Regeneration vor. Danach ist das Bindegewebe, bestimmte einschichtige Epithelien, die Osteoblastenschicht des Periosts und die Zellen des Rete Malpighi zur Regeneration von Substanzverlusten befähigt. Darmschleimhaut, Uterusschleimhaut werden aus den Wachstumszonen der Drüsenschläuche ersetzt. Bei zusammengesetzten Drüsen (Leber, Niere) scheint die Regeneration von den mit kubischem Epithel ausgekleideten, interstitiellen Gallengängen, resp. von den geraden Harnkanälchen auszugehen. Regenerationserseheinungen am Zentralnervensystem, sowie 
den gleichhoch differenzierten Sinnesorganen fehlen bei den höheren Vertebraten, können aber bei den niederen Klassen (Reptilien, Amphibien) studiert werden, und hier läßt sich (nach Schaper) namentlich für das Rückenmark der Amphibien oft noch ein völliger Ersatz verloren gegangener Teile beobachten, der bei erwachsenen Reptilien fehlt oder unvollständig erfolgt.

Die Ausgangsstellen der Regeneration sind wiederum dỉe für die physiologische Regeneration spezifischen Plexusepithelien, die Ependymkeile und die Pseudoepithelien - die Ependymzellen - des Gehirns. Typische Regeneration unter pathologischen Bedingungen wird am erwachsenen und auch am embryonalen Gehirn höherer Wirbeltiere nicht mehr beobachtet (Scha per). Die Ausfüllung von Defekten erfolgt hier durch Bindegewebe und die Neuroglia, und nur für diese könnte ein Erhaltenbleiben ihrer in den Indifferenzzonen gelegenen Bildungsstellen angenommen werden.

Die von Schaper gemachten Erklärungsversuche der Geschwulstentstehung werden unter Betonung des rein spekulativen Charakters seiner Ausführungen gegeben. Später wurden sie u. a. von Versé aufgenommen. Nun sind aber Regeneration und Tumorbildung darin ihrem Wesen nach verschieden, daß die Indifferenzzonen wohl das temporäre in der Bildung eines physiologisch funktionierenden, begrenzten Wachstums im Körper erklären können, keineswegs aber das für die Geschwülste charakteristische, andauernde, prinzipiell zu keiner Bildung physiologisch funktionierender, weil nicht zu Ende differenzierter Zellen führende Wachstum. Abgesehen von dieser Differenz steht bei den physiologischen Prozessen im normalen Zustand und bei der Regeneration die Bildung von spezifischen, normale Produkte liefernden Zellen zur Diskussion, bei den pathologischen Prozessen - die Bildung anormale Produkte liefernder Gewebe.

Das Problem wäre zweifellos leichter angreifbar, wenn man zu einer präzisen Fragestellung kommen könnte. Für die Geschwulstbildung scheint mir an erster Stelle die Frage nach den im Gewebe gegebenen biologischen Wachstumsmöglichkeiten zu stehen, dann die der Auslösung und drittens die der Reaktion, ob begrenzt oder unbegrenzt, und unter dieser Kategorie wäre das Problem der Malignität zu untersuchen.

Wenn nach unseren bisherigen Cntersuchungen die Wachstumspotenz der Gewebe in entwicklungsmechanischen Vorgängen keine Erklärung finden könnte, so liegen erst recht die Frage der Auslösung und die der Reaktion auf anderen Gebieten. Die Wachstumsreize bei der Regeneration scheinen in einer Beziehung wesentlich von denen bei der Geschwulstbildung verschieden. Bei der Regeneration ist formal ein Verlust in erster Linie quantitativer Art $\mathrm{zu}$ ersetzen 
und ein gestörtes Gleichgewicht wieder herzustellen, während bei der Geschwulstbildung formal keine quantitativen Verluste vorliegen. Betrachtèt man aber diese zwei Vorgänge von der qualitativen Seite, so unterscheiden sie sich nur durch den Grad der qualitativen Schädigung. Die Schädigung des Organismus durch Verlust eines Teiles trifft seine Totalität und die Regeneration schafft je nach der Differenziertheit des Organismus den gesetzmäßig denkbar ausgiebigsten, zweckmäßigsten Ersatz, die Schädigung bei der Geschwulst trifft ein bestimmtes System und dieses reagiert, wie im folgenden Absatz auszuführen sein wird, gleichfalls in gesetzmäßiger Form. In beiden Fällen handelt es sich um spezifische Reaktionen, die nach dem Gesetz von Widerstand und Triebkraft quantitativ und qualitativ verschieden verlaufen müssen. Überlegungen der Art, ob die Zellvermehrung bei dem pathologischen Wachstum ein Plus produziert, für das kein Bedürfnis vorliegt (Schaper), daß dadurch unter abnorme Bedingungen zu seiner Umgebung gesetzt ist, bewegen sich im Kreise. Daß dann bei andauernder ,Zellvermehrung infolge des dadurch verursachten Druckes auf die benach barten Elemente, eine Zelle oder Gruppen solcher leicht aus dem Verband ausgestoßen werden, und dauernd den Zusammenhang mit ihrem Mutterboden verlieren“ (Schaper) resp. daß „das umgebende Bindegewebe - durch Druckreiz - in entzündliche Wucherung gerät und hierdurch die Abschnürung von Zellkomplexen aus dem proliferierenden Epithelgebiet begünstigt", also eine umgestellte Ribbertsche Hypothese, sind, abgesehen von den einseitig mechanischen Vorstellungen, biologisch kaum denkbare Möglichkeiten - und vor allem Spekulationen, die in keiner Weise gestützt sind. Die Indifferenzzonen sind in frühesten Entwicklungsperioden am verbreitetsten und nehmen mit dem Alter, d. h. mit fortschreitender Differenzierung gradatim ab. Es muß also, wenn man in ihnen das Ausgangsmaterial zur Geschwulstbildung annehmen will, das entgegengesetzte Verhalten des Organismus erklärt werden, daß Geschwülste in früher Entwicklungsperiode selten und im Alter - dabei noch einem bestimmten Alter - besonders häufig sind. Das Problem ist also unzweifelhaft komplizierter und läßt sich voraussichtlich überhaupt nicht aus diesem einseitig entwicklungsmechanischen Faktor in seiner Totalität erklären.

$\mathrm{Zu}$ seiner Erklärung werden herangezogen: 1. die lange Wachstumsdauer der Tumoren, durch die erklärlich wird, daß die auslösende Schädigung oft $10-20$ Jahre zurückliegen kann, also sehr wohl die Indifferenzzone in einem wachstumstüchtigen Stadium angetroffen haben könnte; 2. daß pathologisches Wachstum im Alter an den Zellen deswegen leichter auslösbar ist, weil dann ihre Zelleneiweiße in einem stabileren Gleichgewicht sind und Schäden damit nicht radikal ausgeglichen werden können. 
M. Vers é ${ }^{42}$ ) hat in seinen beginnenden Carcinomen (tbergänge von normalem Epithel zum undifferenzierten Cylinderepithel und schließlich zu dem atypisch gebauten Krebsepithel beschrieben. Er weist besonders darauf hin, daß ,,mitten in der normalen Schleimhaut veränderte Drüsen auftreten können, deren Umwandlung aus dem vorhandenen Drüsenepithel mit Sicherheit nachweisbar ist". Versé vertritt im Gegensatz zu Ribbert den Standpunkt, daß das Krebsepithel primär umgewandelt ist, und daß, wenn es in die Tiefe vordringt, es rein mechanisch, d. h. dorthin wuchert, wo es den geringsten Widerstand findet, daß das Epithel sich also nicht sekundär, erst nach dem Einwuchern einer rudimentären Drüsenbildung ins Gewebe, verändert. Die von Ribber $t^{32}$ ) angeführte Reaktion in der Umgebung tritt, wie man dieses beim Vordringen einer Neubildung wohl durchweg beim Magendarmearcinom beobachten kann, erst auf, wenn das Carcinom in die Submucosa vorgedrungen ist, wo sich dann in seiner Umgebung Rundzellinfiltrationen resp. bei erfolgter Infektion von außen her Eiterungen finden. Prinzipiell wichtig erscheint, daß diese Infiltration, die im Bingedewebe auch gänzlich fehlen kann, demnach nicht eine Bedingung der Carcinomentwicklung, sondern voraussichtlich eine sekundäre Reaktionserscheinung auf das Carcinomgewebe darstellt. Weder die von Ribbert behauptete vorbereitende Bindegewebsentzündung noch die von ihm angenommene Wegbahnung für das Careinom durch Hypersekretion von Drüsenschleim in die Lymphbahnen brauchen als prädisponierende Momente herangezogen zu werden. Da auch Ribbert zugibt, daß ein Epithel in der Regeneration sich in nichts von dem bei Carcinom unterscheidet, ist eine histologische Diagnose des Charakters solcher Epithelien, wie Versé (l. c.) ausführt, nicht möglich. Er verweist auch in der weiteren Kritik der Ribbertschen Hypothese auf die Schwierigkeiten, die sich für die Erklärung der Charakteränderung des Epithels bei Aufrechterhaltung der Ribbertschen Hypothese ergeben. Drüsenheterotopien, wie sie in den von Versé zitierten Arbeiten R. Me yers ${ }^{25}$ ), von $\mathrm{Lubarsch}{ }^{21}$ ) und von Borst ${ }^{3 a}$ ) beschrieben wurden, sind eben noch lange nicht das Charakteristicum eines Carcinoms. Ob überhaupt rein mechanische Momente bei dessen Entstehung eine primäre Bedingung abgeben, ist nach unseren heutigen Anschauungen sehr zu bezweifeln. Man kommt eben nicht um die primäre Charakteränderung der Epithelzelle herum. Sehr instruktiv für die Selbständigkeit des Epithels ist hier der Fall 44 von Versé.

Die Beobachtungen Versés illustrieren außerdem gut die Kontinuität der benignen und malignen Epithelgeschwülste, die wir neuerdings auch experimentell, bei den Nematoden- und Teercarcinomen dauernd beobachten können. Bei Anwendung dieser experimentellen Methoden operieren wir mit sehr wirksamen, wenn auch noch nicht chemisch 
reinen, d. h. idealen Körpern. Der formale Erfolg einer Geschwulsterzeugung durch Einwirkung bestimmter chemischer Körper auf normale Gewebe ist besonders wertvoll durch die allgemeinbiologischen Gesichtspunkte, die sich aus der Analyse solcher Beobachtungen ableiten lassen. Wir können hierbei zum ersten Mal eine Geschwulstbildung als Resultat des Energieaustausches zweier verschiedener physikalischchemischer Systeme auffassen.

Damit sind die bisher besprochenen entwicklungsmechanischen Momente keineswegs überflüssig geworden, sie behalten ihren Wert als disponierende Faktoren und speziell als Teilbegriff des präcarcinomatösen Stadiums. Sie sind deswegen als relative, eingerichtete Faktoren aufzufassen, weil sie einerseits zu pathologischem, benignem oder malignem Weiterwachstum führen kann, andererseits zum Stillstand oder zur Rückbildung.

\section{Die physikalisch-chemischen Potenzen der reagierenden Systeme.}

Wenn wir davon ausgehen, daß alle Vorgänge in der Natur auf Energiewechsel zurückzuführen sind, ergibt sich die Frage, auf Grund welcher Energien die beteiligten Gewebe beim pathologischen Wachstum reagieren. Da das Protoplasma jeder Zelle ein kolloidales System ist, werden sich seine biologischen Eigenschaften aus der Struktur dieses Systems nachweisen lassen, deren charakteristische Funktionen sie nach der heutigen Auffassung sind.

Der Maßstab der Zuteilung eines dispersen Systems zu den Suspensionen, Kolloiden oder wahren Lösungen ist in der Teilchengröße der dispersen Phase gegeben. Die mikroskopische Vergrößerung ist bei den vollkommensten optischen Systemen begrenzt durch die Beugungserscheinungen, die das hellste Licht an den Teilchen erfährt, und die für unterhalb $0,1 \mu$ liegende Körper nur verwaschene unscharfe Bilder gibt. Feiner disperse Phasen können mikroskopisch daher nur aus der Lichtzerstreuung, die sie bewirken, also indirekt, nachgewiesen werden, resp. - bei Anwendungen kurzwelligerer, als der Lichstsrahlen - mit Röntgenstrahlen. Wenn mit diesen neuerdings an bis dahin für homogen erklärten Körpern Diskontinuitäten nachgewiesen sind, so illustriert dies die willkürliche Fassung des Begriffs ,,kolloider Zustand" resp. ,wahre Lösung". Wenn wir als kolloide Lösungen solche disperse Systeme bezeichnen, wo die Teilchengröße der dispersen Phase zwischen 0,1 " 1,0 "1, liegt, so wird mit diesem Begriff nur ein System bezeichnet, mit dem wir es bei biologischen Cntersuchungen des Protoplasmas vorwiegend zu tun haben. Die wesentliche Eigenschaft dieses Systems ist, daß es kein stabiles ist, sondern einen labilen Zustand bezeichnet. Dicselben Gesetze, die bei den unbelebten Kolloiden - anorganischen und organischen - die Reaktionsweise der Phasen bestimmen, gelten ebenso 
für die Reaktionsweise des lebenden Eiweißes einzelliger und vielzelliger Systeme. D. h. die verschiedenen Zustandsänderungen des Protoplasmas - Viscosität, Sedimentierungsgeschwindigkeit, Kataphorese, osmotischer Druck, Diffusion, Adsorption usw. - sind als gesetzmäßige Funktionen der in den dispersen Phasen gegebenen elektro-chemischen Energiesysteme aufzufassen. Die molekulare Struktur organischer Verbindungen muß dabei letzten Endes ebenso aufgebaut sein, wie die der anorganischen, $d . h$. gegeben sein in der jedesmal typischen Anordnung von Elektronen um einen Kern. Es würden dabei elektrische Ladungen durch die Elektronen den Charakter jedes Moleküls und damit auch jedes Molekülaggregats im Protoplasma bestimmen.

Reagieren demnach zwei oder mehrere solcher Systeme, so muß es zum Austausch der gegenseitigen physikalisch-chemischen - in diesem Falle der elektrischen Energien kommen. Der Maßstab der erfolgten Reaktion, d. h. die Umwandlung im System - läßt sich dann, bei einfachen Versuchsbedingungen, abhängig von dem Grade des erreichten Endzustandes, durch bestimmte physikalisch-chemische Methoden gewinnen. Kommen gröbere Strukturänderungen zustande, so sind diese oft schon makro- oder mikroskopisch ohne weitere Hilfsapparate nachweisbar (verschieden hoher Grad der Ausflockung - von der trüben Schwellung bis zur Nekrose); es können aber auch andererseits Umlagerungen erfolgen, die sich, trotz einer gänzlich abweichenden Struktur und der in ihr gegebenen neu entstandenen Funktion des betreffenden Körpers, nur mit wenigen oder gar nur mit einer einzigen Methode physikalisch-chemisch nachweisen lassen (einfache, Distanz- oder Spiegelbildisomere). Aus der experimentellen Biologie sind nun eine Reihe Tatsachen bekannt, wo sich biologische Prozesse, wie die Entwicklungsanregung, Furchungsanregung, Narkose, Senkungsgeschwindigkeit, willkürlich durch Änderung der physikalisch-chemischen Bedingungen in einem reagierenden System erzielen lassen, und wir sind berechtigt, auf Grund der mitzuteilenden experimentellen Eingriffe anzunehmen, daß sich ebenso das pathologische Wachstum und die Allgemeinschädigung des übrigen Organismus auf ähnliche abgeänderte Bedingungen in einem der reagierenden Systeme zurückführen läßt - daß diese Erscheinungen also Symptome einer abgeänderten inneren Struktur des Protoplasmas sind. Wenn dabei durch die Einführung anorganischer und organischer chemischer Verbindungen, also unbelebter Körper, eine spezifische gut- oder bösartige Neubildung an beliebiger Stelle des geeigneten Substrates (Epithel, Bindegewebe) willkürlich erzeugt werden kann, so heißt das mit anderen Worten, daß durch unbelebte Körper ein biologischer Prozeß eingeleitet worden ist. Analoge Vorgänge sind für das pathologische Wachstum ebenso für die chemischen, unbelebten Stoffwechselprodukte bestimmter Gewebsparasiten anzunehmen, und zwar 
sehen wir hier absichtlich von der sonst üblichen Annahme der primären Wirkung eines belebten Virus oder der von Fermenten ab, weil ihre Annahme im Vergleich zu unserer energetischen Auffassung der Wirkungsweise unbelebter anorganischer oder organischer Verbindungen, sich nicht ohne Erklärung ihrer spezifischen Wirkungsweise verstehen läßt. Diese wird aber letzten Endes auch nur eine physikalisch-chemische sein.

Eine Anwendung der Theorien über die Bildung kolloider Systeme auf das hier behandelte Thema erscheint noch verfrüht. Sie können daher nur in allgemeinen Zügen Erwähnung finden. Die Theorie von Wei$\operatorname{mar} n^{43}$ ) geht von der Krystallbildung aus, bei der der Zustand der Materie bedingt wird durch die Anwesenheit von Krystallisationszentren.

Infolge der gerichteten vektorialen Molekularenergien tritt Oberflächenspannung im Krystall auf. Ihr wirkt die Molekularbewegung im Dispersionsmittel entgegen, dessen Teilchen die Krystallmoleküle der Oberflächenschicht durch ihre Bewegung fortzuschleudern suchen. Es konkurrieren also die dispergierende Energie des Dispersionsmittels und die orientierende der dispersen Phase.

Die Reaktionsenergie nimmt mit dem Dispersitätsgrad zu. Da die feindispersen Teilchen löslicher sind als gröber disperse, kommt es zu einer Lösung, die an großen Krystallen übersättigt ist, und damit zur Kondensation, d. h. zur Bildung von Körpern mit größerer Oberfläche, die sich dann zuerst zu submikroskopischen, dann mikroskopischen Aggregaten zusammenschließen. Der Angelpunkt des ganzen Prozesses liegt danach in der Bildung von Krystallisationszentren, die auf die Annahme gestützt wird, da $B$ die Teilchen sogar einer hochdispersen Phase krystallinischer Natur sind [W. Ostwald $\left.\left.{ }^{28}\right)\right]$.

Nach W. Ost wald ${ }^{28}$ ) handelt es sich beim Dispersionsvorgang um das Freiwerden positiver Oberflächenenergie - also einer Zunahme der Oberflächenspannung, bei übermäßiger Entwicklung von absoluter Oberfläche im Gefolge der Wirkungen expansiver Oberflächenenergie. Jedenfalls muß die Dispersion plötzlich erfolgen. Die hierbei auftretende Gleichgewichtsstörung kann dadurch ausgeglichen werden, daß der Überschuß an freier Oberflächenenergie in eine andere Energieform, z. B. Wärme, umgewandelt wird.

Die Hauptprobleme der Kolloidchemie sind das der Strukturbildung und das der Strukturänderung. Die Bildung kann zustande kommen durch Kondensation mikrostrukturierter oder Dispersion makrostrukturierter Systeme, wie sie in den Theorien von Wei marn ${ }^{43}$ ) resp. Wolfgang Ostwalds ${ }^{28}$ ) gefaßt sind. Da die Kondensation immer von Kondensationszentren (v. Weimarn, l. c.) ausgeht, wird ein verschiedener Dispersitätsgrad resultieren, je nachdem, ob diese Kerne allmählich oder plötzlich eingeführt werden (ungleiche oder gleichmäßige Teilchenbildung). Die Proteine sind augenscheinlich alle durch Kon- 
densation zustande gekommen, jedenfalls spricht von rein chemischem Standpunkte aus der Umstand dafür, daß nach E. Fischer ${ }^{9}$ ) die Eiweiße Kondensationsprodukte sind.

Im Dispersionsvorgang ist nach The S ved ber $\mathrm{g}^{39}$ ) entgegen der Oberflächenenergie und Kohäsionskraft Arbeit zu leisten, daher ist dieser Vorgang kein spontaner, sondern im Gegensatz zum Kondensationsproze $\beta$ ein erzwungener. Falls eine erhöhte Dispersität des kolloidalen Zustandes nachweisbar wäre, könnte sich voraussichtlich aus ihr die erhöhte Wachstumsenergie des ganzen Systems erklären und es könnten sekundär eine Reihe von Erscheinungen verśtändlich werden, wie die erhöhte, lokal auf die pathologischen Zellen beschränkte Assimilationskraft und die herabgesetzte Nahrungsverwertung der übrigen Körperzellen usw. Eine solche Erklärung liegt nach den Untersuchungen Kottmanns ${ }^{20}$ ) am Serum Krebskranker nahe. Es würde sich dabei um Strukturänderungen des Protoplasmas pathologischer maligner Zellen handeln, die fürs erste nur indirekt - aus Veränderungen der pathologischen Serumkolloide wahrscheinlich zu machen waren, die aber noch nicht ausreichend von uns nachgeprüft sind, um sie hier ausführlich zu besprechen. Im Ausgang solcher Veränderung steht auch hier die Energiezufuhr, die nachzuweisen ist und die im folgenden Absatz näher besprochen werden soll.

Die Entwicklungs- oder Wachstumsanregung durch elektrische Umladung der Teilchen illustriert eine mögliche Wirkung, - die indirekte auf die Oberfläche; es läßt sich aber ebenso nachweisen, daß direkte rein chemische Eingriffe in die Struktur - die Funktion des betreffenden Körpers nicht nur anregen, sondern auch, soweit wir dies mit unseren Methoden verfolgen können, gänzlich abändern können. Und zwar auf verschiedene Weise: durch Bildung von metameren oder isomeren Verbindungen, durch Reaktion einzelner Gruppen eines Körpers untereinander, Bildung ionisierter Gruppen, durch Umlagerung in asymmetrisch gebauten Molekülen, resp. Spaltung racemischer Verbindungen durch Fermente und Katalysatoren. Bei diesen letzteren Gruppen muß nur stets ein aktives System vorhanden sein, während die Entstehung seiner Energien noch ungeklärt ist.

Die für die allgemeine Pathologie in erster Linie interessierende biologische Wirkung geht nun auf dieselben Verhältnisse - die Wechselwirkung zwischen asymmetrisch gebauten Körpern zurück. Hierbei muß eine spezifische Bindung an bestimmte Gruppen im Molekül angenommen werden, wie wir sie schematisch aus der Ehrlichschen Hypothese kennen. Die Wirkungsänderung infolge von Änderungen in der chemischen Konstitution ist nach P. Eichwald ${ }^{5}$ ) aus dieser gut ableitbar. Ist die verankernde Gruppe besetzt und dadurch unwirksam, so kann überhaupt keine spezifische Wirkung, trotz Intaktheit der wirkenden 
Gruppe, zustandekommen. Andererseits kann die wirksame chemische Gruppe, durch eine andere als die Ausgangsbindung, an das C-Atom gebunden werden, und es resultiert dann eine völlig abweichende Wirkung. So haben z. B. ,,die Ester der salpetrigen und der Salpetersäure gefäßerweiternde Wirkung (Amylnitrit, Nitroglycerin), ist dagegen die $\mathrm{NO}_{2}$ Gruppe nicht durch $\mathrm{O}$, sondern durch $\mathrm{N}$ an das $\mathrm{C}$-Atom gebunden, wie im Nitrobenzol oder in der Pikrinsäure, so ist die Verbindung ein Blutgift". Ebenso instruktiv ist nach Eichwald die Wirkung von o-Benzoesäuresulfinid (Saccharin) und p-Benzoesäuresulfinid auf den Geschmackssinn, die lähmende Wirkung der Methyljodidgruppe, bei deren Bindung an Alkaloide, wodurch ein dreiwertiges N.Atom in ein fünfwertiges übergeführt wird und damit zwei neue freie Valenzen entstehen.

Der Maßstab des auslösensen Energiefaktors ist also in dessen physikalisch-chemischer Konstitution gegeben. Es läßt sich fürs erste nicht annehmen, - jedenfalls aus den wenigen, aus meinen bisherigen Untersuchungen hervorgehenden Formulierungen der experimentellen Fragestellung - daß er irgendwann in einer ein maligen Schädigung des Organismus gegeben sein wird, sondern es wird sich bei ihm - in Analogie der Gewebsparasiten (Spiropteren), der Teercarcinome, der Anilin- und Röntgenkrebse - um chronisch kontinuierliche Schädigungen handeln, die bei bestimmter kürzerer Einwirkungszeit auf das Versuchstier relativ benigne - bei bestimmter längerer - maligne pathologische Wachstumsprozesse einleiten. Eine Qualitätsänderung der pathologischen Veränderung scheint, wenn sie einmal erreicht ist, aus sich selbst nicht möglich.

Diese Beobachtung fordert zu ihrer Erklärung die Hilfshypothese der Disposition, die von mir bisher einer experimentellen Analyse nicht unterzogen wurde und in ihrer Totalität auch heute noch nicht experimentell angreifbar erscheint. Da aber einzelne ihrer Teilfaktoren -- das Alter, die Röntgenschädigung des lymphoiden Apparates, die Immunitätsprobleme - unserem physikalisch-chemischen Verständnis näher gebracht werden können, erscheint ihre Aufklärung nicht aussichtslos.

Der Dispositionsbegriff, den wir - jedenfalls in seiner Totalität mit unserem heutigen wissenschaftlichen Rüstzeug nicht formulieren können, muß fürs erste noch herhalten, um die Lücken unserer Kenntnisse für die verschiedene Reaktionsform der einzelnen Arten und Individuen notdürftig zu decken.

\section{I.}

Gehen wir nunmehr direkt zur Untersuchung des Energiewertes verschiedener biologischer Systeme über, so muß festgestellt werden, in welchen Körpern die zwei den Energiewert bestimmenden Faktoren - Stoffmenge (Kapazität) und chemische Affinität (Intensität) gegeben 
sind. Die Umwandlungen einer Energieform in eine andere ist eine Funktion der chemischen Affinität der reagierenden Systeme und es wird demnach auch für das Zustandekommen des pathologischen Wachstums zu untersuchen sein, in welcher Form in den betreffenden Systemen der Energiefaktor vorliegt und zweitens, ob, und weiter wie seine Wirkung zustande kommen kann. Diese Fragen wurden bei der biologischen Analyse von Versuchen zu beantworten gesucht, die das Problem des pathologischen Wachstums unter Benutzung verschiedener Energiefaktoren in Angriff nahmen.

In erster Linie wurden die energetischen Bildungsmöglichkeiten von Amphibien und Fischembryonen, die gleich nach Ablage des Laichs oder in den ersten Furchungsstadien implantiert wurden, untersucht. In idealster Weise entsprechen hier der alten Cohnheimschen Theorie die experimentellen Bedingungen Belogolowysi), der durch Reimplantation von Amphibieneiern in erwachsene Tiere der gleichen Art, die Eier unter abnorme, seiner Meinung nach günstigere Entwicklungsbedingungen bringt. Nach seiner Auffassung müßte in diesen unter den neuen Bedingungen ,,parasitierenden" Eiern eine Rückbildung ihrer artspezifischen höher differenzierten Merkmale zu weniger differenzierten embryonalen eintreten, und es würden dann ihre Zellen den unverbrauchten, weil beim Parasitismus unnötigen Energievorrat, neben der Bildung mehr oder weniger typischer embryonaler Organe und Gewebe, durch Bildung atypischer embryonaler Zellen von pathologisch gesteigerter Energie dokumentieren können. Durch Einwuchern dieser pathologischen ,sarkomähnlichen“ Zellen kommt es nach Belogolow y am Wirtsorganismus zu Zerstörung seiner Gewebe und nach 5-6 Monaten zum Tode.

Bei Ausführung unserer Experimente handelte es sich um die Erzielung dieses Endstadiums. Bei ihrem Gelingen mußte sich eine Bestätigung der Cohnheimschen Theorie insofern ergeben, als im eingesetzten Ei mit einem Faktor operiert wird, dessen normale Entwicklungsmöglichkeiten in allen Etappen bekannt und kontrollierbar sind, und der das Verhalten einer isolierten ,,undifferenzierten" Keimausschaltung mit verspäteter Auslösung seiner Wachstumsenergie in übersichtlicher Form nachbildet.

Es war hier also - abgesehen von den interessanten abgearteten Veränderungen im Ei - festzustellen, l. ob sich die von Belogolow y beschriebene Rückdifferenzierung des Implantats nachweisen läßt, 2. welche Reaktionen das plötzlich eingesetzte Energiesystem im Wirtsgewebe auslöst, und 3. von welchen speziellen Faktoren dieses Systems die von Belogolowy angenommene pathologische Geschwulstbildung hervorgerufen wird. Die Aufklärung seiner Wirkungsweise mußte sich dann aus dem Verhalten beider reagierenden Faktoren ergeben. Ich teile 
hier zuerst unsere Befunde mit, weil anzunehmen ist, daß unter gleichen experimentellen Bedingungen bezüglich Material, Methode der Implantation und der endgültigen Untersuchung des Objekts, sich gleiche Veränderungen des biologischen Verhaltens ergeben müßten. Zur Implantation benutze ich Frosch- und Kröteneier, die 1-2 mal 24 Stunden alt waren. Sie wurden in 0,65 proz. Kochsalzlösung erst a bgespült, danach die äußere Gallerthülle mit sterilem Instrument entfernt und zu 3-5 Exemplaren durch eine Schnittöffnung der Bauchdecken in die Peritonealhöhle implantiert. Die Wunde wurde durch Naht geschlossen, die Tiere kamen dann zuerst in ein Terrarium, nach 24 Stunden ins Aquarium. Um eine Infektion durch Mitübertragung von Mikroorganismen zu kontrollieren, wurden die Gallerthüllen regelmäßig bakteriologisch untersucht. Es fanden sich in ihnen zahlreiche bewegliche Stäbchen und Sproßpilze (des Teichwassers), die sich auch später im mikroskopischen Präparat in der äußeren Zone der Amphibien und Fischeier nachweisen ließen. Makroskopisch war dabei eine Peritonitis nicht feststellbar, abgesehen von spärlichen lockeren Adhäsionen, die zum Teil zwischen Ei und Mesenterium resp. Ei und Darm oder Leber oder zwischen Nahtstelle und Abdominalorganen bestanden. Die Amphibieneier zeigten im mikroskopischen Bild, daß trotz Lupenkontrolle bei Entfernung der Gallerthülle nicht nur deren innere feine Haut vorhanden war, sondern da $\beta$ in allen bis zum Schlu $\beta$ am Leben gebliebenen Exemplaren eine oft den Durchmesser des Eis übertreffende neue äußere Gallerthülle gebildet war.

Eine zweite Serie von Amphibieneiern wurde durch hypertonische 2 proz. NaCl-Lösung oder durch Röntgenbestrahlung (Apex [R. G. u. Sehall] Induktor, Müller S. R. Filter $3 \mathrm{~mm}$ Al. $27 \mathrm{~cm} \mathrm{F.} \mathrm{H.} \mathrm{A.,} \mathrm{Milliamp.}$ $2^{1} / 2$. Dauer 1 Min. $=1 / 1$ H. E. D.) sensibilisiert und dann implantiert. Von Fischeiern wurden je drei Forelleneier (2-6 Tage alt) implantiert, bei diesen konnte die derbe Kapsel ohne schwere Schädigung des Eies mechanisch nicht entfernt werden, sie wuchsen aber trotzdem im homologen Organismus gut an. Von allen Versuchstieren starben in den ersten 3 Wochen $7,5 \%$, die übrigen überstanden den Eingriff äußerlich gut und gingen erst nach 4-6 Monaten zugrunde resp. wurden nach Ablauf von 5 Monaten getötet. Zur Anheilung an Organe des Wirtstieres kam es in $60 \%$, wobei Amphibieneier in erwachsenen Amphibien (Rana temporaria, Pelob. fuscus), Fischeier in Forellen und Karpfen implantiert waren, während Fischeier im Froschorganismus in keinem Fall zum Anwuchs gekommen waren.

Bei der Autopsie ließ sich in keinem Falle nachweisen, daß die Tiere an dem Umfang einer entstandenen Neubildung direkt zugrunde gegangen waren. Die implantierten Forelleneier ließen sich regelmäßig, auch wenn sie nicht angewachsen waren, bei der Autopsie auffinden, während 
die Amphibieneier in den nicht angeheilten Fällen überhaupt nicht nach weisbar waren.

Bei Untersuchung mit schwacher Vergrößerung (Leitz, Ok. 2, Obj. A) sieht man das $\mathbf{E i}$, umgeben von seinen versehieden breiten Gallerthüllen. Es ist entweder mit dem Wirtskörper durch einen schmalen, bindegewebigen Stiel verbunden (H.-J. Nr. 248), oder ist ihm direkt an- oder eingelagert und wird von ihm durch eine zirkuläre Kapsel umschlossen (H..J. Nr. 137, 167, 182, 193, 248). Die Gallerthüllen unserer Amphibieneier lassen eine innere schmale, strukturlose Schicht und häufig, dieser nach außen anliegend, eine breite, kernarme, locker strukturierte Hülle erkennen, die wiederum nach außen von einer zweiten, breiteren strukturlosen Zone umsehlossen wird (H.-J. Nr. 248, 193), die an den hier mehr oder weniger veränderten Wirtskörper grenzt. - Bei stärkerer mikroskopischer Vergrößerung (Leitz Ok. 2, Obj. E) läßt sich nachweisen, daß der inneren Gallerthülle eine einfache Lage großer polygonaler Zellen mit blasigen hellen Kernen anliegt, und daß die äußere strukturlose Gallerthülle ebenfalls von einer $1-2$ fachen Schicht kleinerer, mehr kubischer Zellen mit chromatinreichen dichten Kernen nach außen begrenzt ist. Die zwischen diesen 2 strukturlosen Hüllen liegende lockere Gallertmasse ist verschieden stark entwickelt, enthält bei dieser Vergrößerung im Gesichtsfeld ca. 20-30 runde, kleine, bläschenförmige, chromatinarme Kerne ziemlich gleichmäßig über die ganze Masse verteilt (H.-J. Nr. 248) und ist oft dicht durchsetzt von polymorphen Zellen mit verschieden großen und verschieden strukturierten Kernen, die das Grundgewebe völlig verdecken können (H.-J. Nr. 193), so daß scheinbar ein direkter Übergang dieses Gewebes in das Gewebe des Wirtskörpers besteht. In anderen Fällen sind die Kerne der lockeren Hülle geschwunden, das Grundgewebe ist homogenisiert und zeigt nur spärliche kleine Einschlüsse, die aus eben sichtbaren Zellschatten und zahlreichen dichtgelagerten, runden Chromatinpartikeln bestehen und dann gegen die Umgebung durch eine zarte Schichtung der Grenzsubstanz abgegrenzt sind. Außerdem finden sich in der äußersten Gallertzone dichte Kolonien von Mikroorganismen, die zentralwärts stark an Masse abnehmen und in einzelnen Fällen zu kleinen rundlichen Herden zusammengeschlossen sind, wiederum mit einer sekundären feinen Grenzschicht gegen das Gallertgewebe (H.-J. Nr. 167). In der nächsten Umgebung - vor allem im Gewebe des Wirtskörpers - finden sich in solchen Fällen besonders reichlich Zellen von polynucleärem Typus. Die Gallerthüllen sind besonders mächtig um Eier, die bis zum Schlusse gelebt hatten, sind dagegen schwach um Eier, die tot waren und können hier, bis auf die innere schmale, strukturlose Hülle, nur eben angedeutet sein.

Eier, die nur in den ersten Wochen nach der Implantation gelebt haben, zeigen ungleiche Blastomeren, mit einem oder mehreren verschieden großen, meist dichten Kernen, die in den zentralen Partien weniger, in den peripheren reichlich Dotterzellen enthalten (H.-J. Nr. 167). Da Zellgrenzen an einzelnen kernreichen Blastomeren oft nicht nachweisbar sind, liegen hier scheinbar syncytiale Bildungen vor. - Die Ausbildung der Keimblätter und die weitere Entwickelung des Eies bis zur Bildung von Organanlagen erfolgt verspätet und äußerst u ?gleichmäßig. Es werden dabei meist nur einzelne Organe der betreffenden Entwickelungsstufe angelegt - Chorda, Gehirnblasen -, während die übrigen zu diesem Stadium gehörigen Bildungen gänzlich fehlen können. Außer diesen Bildungen finden sich regelmäßig in der Eiperipherie kleine rundliche, oder etwas unregelmäßige, homogen erscheinende Nuclearpartikel, die an die äußere Gallertmembran gepreßt erscheinen und häufig von dichteren Pigmentniederschlägen umhüllt sind. Außer diesen physiologischen Bildungen lassen sich keinerlei andere Körper, vor allem nicht ,selbständig gewordene " embryonale Zellen in kleineren oder größeren Verbänden im 
Ei nachweisen. Sollten - nach Belogolow ys Auffassung - selbständig gewordene embryonale Zellen in die Wirtsorgane einwandern, um diese nicht nur zu infiltrieren, sondern auch zu zerstören, so müßten sie auf ihrem Wege die Gallerthüllen passieren und in ihnen sich nachweisen lassen. Dieser Nachweis ist aber weder von Belogolowy erbracht, noch ist er uns - aus später zu besprechenden verständlichen Gründen - gelungen.

Wenn das Ei am Mesenterium inseriert ist, so sind hier in dessen lockerem Gewebe außer dem für die Ausbreitung des Granulationsgewebes günstigen, lockeren Boden scheinbar auch bessere Bedingungen für das Wachstum der mitübertragenen Mikroorganismen gegeben und das Granulationsgewebe nimmt dann einen relativ großen Raum ein. Bemerkenswert erscheint nebenbei, daß obligate Parasiten des Frosches, wenn sie sich in den Präparaten am Darm oder am Mesenterium angewachsen finden, im Vergleich zu dem Ei eine wesentlich geringere Reaktion von seiten des Wirtskörpers hervorrufen - voraussichtlich, weil sie keinerlei Mikroorganismen mit sich führen, die körperfremd wie die Mikroorganismen des Teichwassers sind (H.-J. Nr. 207, 248).

Wenn sich nach unseren Beobachtungen die das maligne Wachstum auslösenden embryonalen Zellen Belogolowys nicht nachweisen lassen, so fragt es sich weiter, ob nicht die ganze von Belogolow y als Sarkomgewebe aufgefaßte Bildung ebenfalls anders bewertet werden muß. Zur Beurteilung seiner Schlüsse liegt Material vor allem in seinen Handzeichnungen vor. Die Zeichnungen Taf. 27, Abb. 37, Taf. 28, Abb. 42, 43, Taf. 29, Abb. 59, 60, Taf. 31, Abb. 71, 72, 73 sind, als Wiedergaben von Gewebsausschnitten, dabei die einzigen, die für einen Beleg in Betracht kommen könnten. Von den Mikrophotographien läßt sich keine als Beleg der Bildung pathologischer bösartiger Geschwülste verwerten. Aber auch in den Handzeichnungen zeigen nur besonders Abb. 42, 72 Zellen mit relativ massigem Protoplasma, aber kleinem Kern, die frei in Bindegewebsmaschen liegen. Durch keine aus den Zeichnungen erkennbare Eigenschaft dieses Gewebes läßt sich aber sein Charakter als pathologisch bösartige Neubildung erweisen. Daß hier der unbestimmte Ausdruck Belogolow ys ,,sarkomähnlich" durch den eine präzise Vorstellung gebenden und diese wohl auch anstrebenden des ,Sarkoms" zu ersetzen ist, hoffe ich im Sinne des Autors getan zu haben. Zur Erklärung der Frage nach den Qualitäten dieser Hauptstütze der Belogolow yschen Hypothese sind wir also, um eine gemeinsame Basis der Beurteilung zu schaffen, auf den Vergleich dieser Bilder mit unseren Präparaten angewiesen und diese zeigen folgendes:

An die äußere Grenzschicht der Gallerthülle grenzt eine, je nach der Struktur des anliegenden Wirtsorgans breitere oder schmälere Zone von zentral engerem, peripher lockerem Maschenwerk aus jungen Bindegewebszellen. In diesen Maschen liegen polymorphe Zellen mit meist blassem, nach Giemsa blaugefärbtem, nach Unna-Pappenhei m rotgefärbtem, granuliertem Protoplasma, mit verschiedengeformten und verschiedengearteten Einschlüssen; das Gewebe ist reich an Blutgefäßen, zeigt einige freie Pigmentkörner und freie Erythrocyten und Leucocyten. Die in den Maschen liegenden Zellen sind, sowohl nach den Zeichnungen Belogolowys, als auch nach unseren Präparaten, nirgends zu Geweben zusammen- 
geschlossen, und zeichnen sich hauptsächlich durch die verschiedene Färbbarkeit ihres Protoplasmas, ihrer Granula, ihrer Kerne und der übrigen Protoplasmaeinschlüsse: Erythrocyten, Chromatinpartikel, Vakuolen und Dotterzellen aus. In dem Maschenwerke liegen außerdem einzelne Riesenzellen von Langhansschem Typus mit 10-40-80 Kernen. Nach färberischem und morphologischem Verhalten sind die vorliegenden Zellen zum Teil losgelöste Endothelien, große und kleine lymphoide Zellen, groß- und kleinkernige, fast ungranulierte resp. baso- und eosinophile Granulocyten, Histiocyten und Plasmazellen.

Die Zellen sind eindeutig als typische Bestandteile eines Granulationsgewebes zu bestimmen und es besteht in keinem Fall, schon aus der Kernplasmarelation und aus dem isolierten, nicht zu Geweben zusammengeschlossenen Verhalten der Zellen, eine Möglichkeit, in ihnen Zellen eines Sarkomgewebes anzunehmen. Nach unseren Befunden haben wir ein typisches Granulationsgewebe, also nicht das Resultat einer aktiven Infiltration mit Tumorzellen, die vom Implantat ihren Ausgang nehmen, sondern umgekehrt das Resultat einer aktiven Reaktion des Wirtsorganismus a uf das Implantat vor uns. Dieser Auffassung nach ist dann das Postulat des Nachweises einer Durchwanderung der embryonalen Zellen vom Implantat in den Wirtskörper nicht mehr nötig, weil es keine embryonalen Zellen sind, und damit erscheint vor allem die Theorie Belogolow ys von der Einwirkung parasitären Lebens auf die implantierte embryonale Zelle nicht haltbar.

Unsere bisher nicht erwähnten Resultate der Implantation späterer Entwicklungsstadien resp. sensibilisierter Eier geben für die Gesamtfrage nichts prinzipiell Neues, da sie sich sowohl nach Lebensdauer wie nach Weiterentwicklung völlig parallel den frühen Stadien verhalten. Wenn - nach unseren Beobachtungen - sich die Belogolow yschen theoretischen Postulate nicht bestätigen lassen, so liegt das nicht nur an einer verschiedenen Deutung des prinzipiell gleichen Materials, sondern an der im Ausgangspunkt gegebenen Wertung der Bildungsmöglichkeiten des Implantats, die zur Lösung des Problems ungeeignet ist. Das pathologische Wachstum ist überhaupt kein Form problem, sondern ein Problem der Substanz. Wenn wir in der Embryologie bestimmte Formbildungen unterscheiden, und dabei noch eine folgende auf die zeitlich frühere ursächlich zurückführen, so ist dies jedenfalls nur aus praktischen Einteilungsgründen zulässig.

Belogolowy ist von der unbestreitbaren Tatsache ausgegangen, daß frühere Bildungen eines Embryo wesentlich einfachere Formen zeigen als spätere; beide sind durch das gemeinsame Band der Formkontinuität verknüpft. In der früheren Form ist also nach seiner evolutionistischen Auffassung die spätere in nuce enthalten. Das Material zur Bildung späterer komplizierterer Formen müßte dann in potentieller Energie in der früheren Form gegeben sein. Kommt es zu einer Rückbildung des unter den neuen parasitären Bedingungen ,,unnötig“" ge- 
wordenen Arteharakters, so stellt sich Belogolow y vor, daB der theoretisch annehmbare Utberschu $B$ an Formenergie nun in einer anderen Energieart investiert wird - und zwar als pathologisches aggressives Wachstum. Aus sich selbst heraus kann wohl ein solches nicht entstehen. sondern es muß ein Faktor von außen hinzutreten, der den Prozeß in Bewegung setzt. Der Faktor, der auf das besprochene experimentelle Implantat einwirkt, ist das veränderte Milieu; nach dem Resultat der Experimente können diese Milie ubeding unge $n$ aber nur in besti m mter Richtung wirkend angenommen werden, und zwar muß das Stehenbleiben auf einem Entwicklungsstadium resp. die verlangsamte Weiterentwicklung, bei dem Mangel irgendwelcher anderer Faktoren auf die experimentelle Schädigung durch die abgeänderten Stoffwechselbedingungen und - vielleicht noch auf die Einbringung von Mikroorganismen - zurückgeführt werden. Ob hier neben der Verringerung oder gänzlichen Drosselung der O-Zufuhr noch andere Bedingungen im Wirtsorganismus gegeben sind, ist für unsere Fälle im Augenblick von sekundärer Bedeutung. Aus den Resultaten des Experiments kann m. E. nur geschlossen werden, daß unter den willkürlich geschaffenen Bedingungen das Implantat trotz des artgleichen Bodens 1. nicht ausreichend ernährt wird, 2. nicht rückdifferenziert wird, sondern entweder abstirbt oder nur mühselig sich unter den ungünstigen Bedingungen weiterentwickelt. DaB diese Weiterentwicklung nicht mit einer gleichzeitigen Energiespeicherung verbunden ist, geht mit aller wünschenswerten Deutlichkeit daraus hervor, daß das Implantat vom Wirtskörper wie jeder beliebige Fremdkörper eingesehlossen und abgebaut wird.

Die experimentellen Bedingungen genügen jedenfalls nicht, um das Implantat zu pathologischem Wachstum anzuregen, weil sie scheinbar nur die Erhaltungsbedingungen der energetischen Struktur. nicht aberderen innere Konstitution ändern. Eine Änderung der Funktion eines kolloidalen Systems wie des Protoplasmas kann aber nur erfolgen nach Umbau seiner energetischen Struktur. und hierzu ist allem Anschein nach die Zufuhr eines neuen, von außen hereingebrachten Energiesystems erforderlich.

Es würde demnach weder für die abgesprengten embryonalen Zellen Cohnheims, noch für das isolierte, korrelativ gebundene Energiesystem der Eizelle, die autonome Bildung einer abgeänderten pathologischen Funktion aus der in ihnen entwicklungsmechanisch gebundenen Formenergie zu erwarten sein. Für die Auslösung solch eines Prozesses muß das Eingreifen eines Systems mit freier Energie gefordert werden.

II.

Ein von diesen ,,undifferenzierten" Zellen wesentlich abweichendes Energiesystem haben wir in den transplantablen Mäusetumoren. Der 
Impferfolg ist bei den verschiedenen verimpfbaren Tumoren wechselnd und nur selten haben wir Stämme, die dauernd 100\% Erfolg geben. Die meisten wachsen subkutan, bilden lokalisierte, locker im Gewebe fixierte Tumoren, die nur ausnahmsweise einen malignen Charakter zeigen, $d . h$. infiltrativ wachsen resp. metastasieren. Bei einem einzigen von unseren Stämmen, der intramuskulär wuchs, einem Riesenzellencarcinomsarkom, das ich Herrn Privatdozent Dr. Fr. Ke ysser - Jena verdanke, wurde Arrosion des Knochens beobachtet ${ }^{44}$ ). Es gelang, diesen Tumor auch subkutan weiter zu züchten, aber dabei ging sein früherer maligner Charakter verloren. Alle diese Umstände kennzeichnen die Tumoren als experimentelle Metastasen, bei denen eine bestimmte begrenzte Wachstumsenergie mittransplantiert wird, und denen das maligne Wachstum meist fehlt. Es liegt nahe, daß solche Tumoren durch bestimmte Erreger hervorgerufen werden, die die Zellen des Transplantats zu einer begrenzten Vermehrung veranlassen, danach sich aber erschöpfen. Die Annahme eines äußeren Momentes von der Art eines ultravisiblen Virus, würde auch den Umstand erklären, daß in gewissen Fällen nicht eine Geschwulst vom Typus eines Carcinoms, sondern eines Sarkoms entsteht. Diese Tumoren eignen sich aber für das Studium der Tumorpathogenese so lange nicht, als der aktivierende Körper (z. B. das Virus) noch unbekannt ist; sie sind außerdem aus dem Grunde ungeeignet, weil sie nur ausnahmsweise infiltrativ und metastasierend wachsen; der schwerstwiegende Grund für ihre Unzulänglichkeit bei unserer Fragestellung ist aber darin gegeben, daß bei diesen Tumoren überhaupt nicht ihre Entstehungsweise beobachtet werden kann, weil sie willkürlich, im Stadium vollster Entwicklung, transplantiert werden, wo meist nur das exzentrische Wachstum ganzer Zellkomplexe allein beobachtet werden kann.

Weiterverimpfungen der sporadisch bei unseren Mäusen zur Beobachtung gelangten Spontantumoren gelangen uns nur durch zwei, höchstens drei Generationen. Die Transplantate gingen trotz der üblichen Kautelen immer langsamer an, wurden vom Lymphocyten infiltriert, zeigten zentralen Zerfall und wurden schließlich gänzlich resorbiert. Ausgehend von der Beobachtung ${ }^{27}$ ), da $\beta$ die Immunität in einem Teil dieser Fälle morphologisch mit einer reichlichen Ansammlung von Lymphocyten in der Umgebung des Transplantats verbunden war, und sich auch hämatologisch dabei eine absolute Zunahme der Lymphocyten nachweisen ließ, suchten wir dies Phänomen näher zu klären. Durch täglich wiederholte Bestrahlungen der zukünftigen Implantationsstelle an weißen Mäusen in einem Ausmaß von $1,5 \times 1,5 \mathrm{~cm}$ (Veifa. Intensivreformapparat, Coolidgeröhre, 21 Kilovolt, 2 Milliamp.-Funkenstrecke $20 \mathrm{~cm}$, ohne Filter, H. A. $25 \mathrm{~cm}$, Tubus $6 \times 8 \mathrm{~cm}, 5$ Min. Dauer $=17,5$ Fürstenau) sollte die lymphocytäre Reaktion aufgehoben werden. Dies 
gelang aber, wie an anderer Stelle berichtet werden wird, im Gegensatz zu den Mitteilungen Murphys ${ }^{27}$ ) und seiner Mitarbeiter keineswegs regelmäßig. Man erzielt wohl in solchen Fällen einen vorübergehenden Sturz der Lymphocyten von ca. 12-20000 auf 2-3000, aber diese Senkung des Lymphocytenniveaus hält nur 8-10 Tage an und genügt meist nicht, um das Angehen der Tumoren prinzipiell zu garantieren. Das Verhalten der Lymphocyten ist für das Problem der Immunisierung gegen experimentelle Mäusetumoren theoretisch beachtenswert, reicht aber nach unseren Beobachtungen für die angestrebte Klärung der Immunitätsbedingungen nicht aus.

Zufriedenstellende Erfolge hatten wir, abgesehen von den später zu erwähnenden experimentell erzeugten Carcinomen, mit dem Keysser schen Riesenzellensarcokarcinom, das bei Verimpfung seiner Tumoremulsion in die Muskulatur des Oberschenkels in 100\% angeht. Es ist ausgezeichnet durch stark infiltrierendes Wachstum in der Muskulatur, zerstört diese und kann in den Knochen einwuchern. Die Tumoren gehen bis ca. zum 10. bis 12. Tag nach der Verimpfung ausgezeichnet an, wachsen bis Kirschgröße, zerfallen dann durch plurizentrische zentrale Einschmelzung und werden bis auf geringe Reste, die meist nur aus einem lockeren Fett- und Bindegewebe bestehen, resorbiert. Auch diese Tumoren ließen sich nicht durch Röntgenvorbehandlung der Wirtstiere zu energischerem quantitativem und qualitativem Wachstum bringen. In der Peripherie fanden sich nur spärliche Lymphocyten, in den zentralen Einschmelzungen polymorphkernige, neutrophile Leukocyten. Im Unterschied zu den Spontantumoren besitzen sie jedenfalls eine erhöhte Malignität, die sich auch durch die fehlende bindegewebige Abkapselung des Implantats dokumentiert. Metastasen haben wir bisher nicht beobachtet. Da die Tumoremulsion nach Passage durch Berkefeld und Chamberlandfilter, ebenso nach energischem Zerreiben mit Kieselguhr unwirksam ist, muß für diesen Tumor angenommen werden, da $\beta$ sein Angehen an das Vorhandensein intakter Zellen gebunden ist. Es handelt sich danach um direkte Transplantationstumoren, deren zeitlich bestimmte Wachstumsenergie mit endgültiger Resorptionstendenz und die fehlende Metastasenbildung, sie prinzipiell gegenüber den Rou xschen Tumoren auszeichnet. Auf die Pathogenese dieser letzteren kann hier mangels eigenen Materials nicht eingegangen werden. Aber wenn auch, wie zu erhoffen ist, die neuerdings von Teutschlä nder ${ }^{10}$ ) gemachten näheren Bestimmungsversuche der Virusart erfolgreich sein sollten, hätten wir eine Vorstell ung von der Art des a uslösenden Faktors gewonnen, nicht aber eine Vorstellung von dem Wesen seiner Wirkung. 
III.

Die zuletzt erwähnten Untersuchungen über die Rolle eines ultravisiblen Virus in der Pathogenese maligner Tumoren gehören, der Kategorie des wirksamen Faktors nach, in das Gebiet der Gewebs- resp. Zellparasiten, die malignes Wachstum auslösen können. Klinisch sind eine Reihe solcher Parasiten: Bilharzia, Distomum felineum, Trichinen u. a. bekannt, experimentell gelang es zuerst Fibig er ${ }^{6}$ ), durch Verfütterung nematodenhaltiger Schaben an bunte Ratten, bei diesen in über $\mathbf{5 0} \%$ ein Carcinom des Vormagens zu erzeugen. Diese Nematoden (Spiroptera neoplastica) sind obligate Gewebsparasiten, die sich unter normalen Bedingungen der Wirtstiere in bestimmten Tierarten - Ratten und Mäusen - - und bei diesen wieder nur an bestimmten Stellen im Plattenepithel - (Zungengrund, Oesophagus, Vormagen, Vagina) ansiedeln. Wenn es Fibiger bei weißen Mäusen bisher nur in $3 \%$ gelungen ist, eine Krebsbildung zu erzeugen, und dies nur bei durch Alter und Inzucht ,disponierten" Tieren, so besagt dies, daß die Spiropteren einen spezifisch geeigneten Nährboden brauchen, erstens, um selbst zu existieren, und zweitens, um die maligne Änderung der betreffenden Zellstruktur zu erzielen. Daß aber hierzu ganz spezifische Strukturen vorliegen müssen, geht daraus hervor, daß bei Ansiedlung der Parasiten in dem morphologisch gleichen Epithel des Oesophagus oder im Epithel der Vagina bunter Ratten nie Carcinombildung beobachtet ist. Bemerkenswert erscheint zweitens, daß die Parasiten, nach Auslösung des malignen Wachstums, lokal fehlen können und in den Metastasen nie angetroffen wurden, daß sie den Prozeß also nur einleiten. Für die Art der Wirkung kommt in Betracht, daß der Parasit kontinuierlich, und zwar eine bestimmte minimale Zeit (bei der Ratte mindestens 45 Tage) einwirken muß, und daß hierbei zuerst die Krebsbildung durch eine Etappe morphologisch scheinbar gutartiger Epithelhyperplasien und Papillome - das präkanzeröse Stadium - geht. Ein zwangsläufiger Zusammenhang zwischen diesen morphologisch ,gutartigen" Bildungen und der Krebsentwicklung besteht jedoch nicht. Diese experimentelle Beobachtung deckt sich also völlig mit den Orthschen Beobachtungen an menschlichem Material ${ }^{29}$ ). Das in unserem Sinne wertvollste Resultat aber ist das Faktum, daß - zwar bei, wie wir annehmen müssen, nach Art und Funktion spezifisch disponierten Zellrassen - es normal funktionierende Strukturen sind, die zu pathologisch funktionierenden umgebaut werden. Das Rattencarcinom entspricht dabei nach seinem lokalen Wachstum und nach seiner Metastasenbildung prinzipiell den gleichen Eigenschaften menschlicher Carcinome.

So glänzend dieser formale Erfolg der experimentellen Krebserzeugung ist, war es bisher nicht möglich, aus dem Material Vorstellungen 
über das Wesen der Entstehung des malignen Wachstums zu gewinnen. Dafür müßten vor allem die Stoffwechselprodukte der Parasiten - z. B. der Spiropteren - isoliert sein und man könnte dann mit diesen resp. ihren einzelnen Bestandteilen experimentell vorgehen. Unsere Versuche, die Dissimilationsprodukte $\mathrm{zu}$ isolieren, sind bisher ohne Erfolg geblieben. Daß der auslösende Faktor nur in den Spiropteren gebildet werden muß, geht auch daraus hervor, daß gleichzeitig mit ihm im Vormagenepithel lebende Parasiten, wie die von Fibiger ${ }^{6}$ ) und Wassink (zit. bei Fibiger) beobachteten Trichosomen, nie krebsbildend wirken.

Für das Zustandekommen der ,,malignen“ Strukturänderung ist also auch hier die Einwirkung eines bestimmten Aktivators nötig, der in den Stoffwechselprodukten des Parasiten gegeben sein muß.

\section{IV.}

Es war demnach eine Methode der Krebserzeugung zu suchen, bei deren Anwendung die reagierenden Komponenten sich nach Konstitution und nach Ubersehbarkeit ihrer Wirkung klarer fassen ließen. Ideale Bedingungen wären, wie ich an anderer Stelle ${ }^{2}$ ) theoretisch ausführte, gegeben, wenn man den Reizfaktor physikalisch und chemisch definieren und seine Lokalisation am Wirkungsort wenigstens nach dem Typus der qualitativen Analyse einwandfrei nachweisen könnte. Die Erfüllung dieses damals theoretischen Postulats ist, soweit unsere Untersuchungen bisher gediehen sind, scheinbar näher gerückt bei den Teercarcinomen und wird sich außerdem vielleicht dann mit physikalisch exakten Methoden durchführen lassen bei experimentellen Röntgencarcinomen.

Die Teercarcinome sind experimentelle Nachbildungen der bekannten Schädigungen bei Arbeitern der Steinkohlenindustrie. Zählt man dieser sowohl die Betriebe zu, in denen aus der Kohle die ersten Destillationsprodukte: Steinkohlenteer, Pech, Rohparaffin, Rohpetroleum, Schmieröle resp. aus diesen durch mechanische Verarbeitung mit anderen Abfallstoffen der Industrie, z. B. Briketts gewonnen werden, als auch die Industrien oder Betriebe, in denen der Arbeiter entweder mit diesen Produkten dauernd in direktem Kontakt ist oder bei der industriellen Weiterverarbeitung der Destillationsprodukte (Substitutionen am Benzolkern, wie bei den Toluolen, Xylolen, Phenolen - resp. in der Farbindustrie - Aminen (Anilinen usw.), Nitroprodukten (Nitrobenzolen usw.) den Dämpfen dauernd ausgesetzt ist, so ergibt sich eine kaum übersehbare Reihe chemisch meist gut definierbarer Körper, die für die Auslösung des malignen Wachstums verantwortlich gemacht werden.

Beobachtet sind solche Industrieschädigungen durch direkten Kontakt an den äußeren Hautdecken, durch Einatmung von Dämpfen in 
der Anilinindustrie an der Schleimhaut der Blase; die Schädigung des Blutes kommt für die hier diskutierte Frage nicht direkt in Betracht.

Am übersichtlichsten sind die Veränderungen, die an den äußeren Hautdecken auftreten, und diese lassen sich im Experiment gut nachbilden. Solche Experimente wurden zuerst von Ja magiwa und Isch i $\mathrm{kava}^{18}$ ) am Kaninchenohr ausgeführt, das alle 2-3 Tage mit Steinkohlenteer gepinselt wurde. 1918 teilte $\mathrm{H}$. Tsutsui ${ }^{41}$ ) mit, daß er mit Teerpinselung der Haut weißer Mäuse ebenfalls im Endresultat von 67 so behandelten Tieren 16 Carcinome, 1 Sarkom erzielt hatte.

Diese letzteren Experimente wurden dann von Fibiger in Kopenhagen ${ }^{7},{ }^{8}$ ) und von uns in beiden Fällen an 25 Mäusen fortgeführt. Die Fibigerschen Experimente ergaben dabei an 24 von 26 Mäusen, die länger als 180 Tage mit Teerpinselung behandelt waren, die Bildung maligner Tumoren, während in unseren, bis jetzt kurzfristigeren Experimenten bisher maligne Tumoren in $60 \%$ zur Beobachtung gekommen sind. Im Endresultat wurde von Fibiger bei genügend langer Teerbehandlung demnach in nahezu allen Fällen die Bildung einer bösartigen Geschwulst erhalten, die charakterisiert ist lokal durch infiltrierendes, destruierendes Tiefenwachstum, durch Metastasenbildung und die Möglichkeit, den Tumor durch Transplantation weiter zu verimpfen.

Die Methode ist zum Studium der Pathogenese bösartiger Geschwülste in vielfacher Hinsicht ausgezeichnet, denn 1. läßt sich die Tumorbildung an der Haut dauernd makroskopisch verfolgen, 2. ist in dem Teer ein chemisch relativ gut definierbarer Körper gegeben, der wesentlich eindeutiger ist, als die Vielseitigkeit der bei den Spiropteren in Betracht kommenden Stoff wechselprodukte, 3. scheint es möglich, daß der auslösende Faktor auch mikroskopisch an der Reaktionsstelle nachgewiesen werden kann.

Diese experimentelle Methode ist außerdem besonders aus dem Grunde wertvoll, weil sie zeigt, daß für die Entstehung des Tumors in diesem Falle keine belebten Organismen nötig sind, sondern daß auch ein rein chemischer Körper den gleichen Endeffekt haben kann, daß also zwei, ihrer Konstitution nach weit auseinanderstehende Körper den gleichen Effekt haben können. Durch Klärung des Wesens der Teerwirkung scheint daher eine der gewiß vielseitigen Bildungsmöglichkeiten maligner Tumoren aufklärbar, denn, lassen sich überhaupt einmal die physikalisch-chemisch wirksamen Energien definieren, so kann zweifellos die ganze, im Zielpunkt stehende biologische Erscheinung auf übersichtliche Strukturreaktionen der beteiligten Systeme zurückgeführt werden.

Da wir bei den Teercarcinomen beobachten können, daß von ein und demselben Faktor sowohl eine epitheliale, wie eine gemischte 
epitheliale und bindegewebige, schließlich, im Tsutsuischen Fall, eine rein bindegewebige maligne Neubildung erzielt werden kann, erscheint die Annahme möglich, daß der auslösende Faktor nicht spezifisch auf eine bestimmte Zellart eingestellt zu sein braucht, daß er vielmehr als allgemeiner, nur relativ spezifischer Faktor - sowohl die eine Gewebsart, wie die andere - resp. beide durch eine ihnen gemeinsame Komponente, zu pathologischen Bildungen veranlassen kann. Wesentlich scheint dabei, daß es eine von außen zugeführte freie Energie ist, die das hochdifferenzierte, aber korrelativ gebundene Energiesystem der normalen Struktur in ein pathologisch funktionierendes umbaut. Diese freie Energie, die, wie wir im letzten Abschnitt sehen werden, erforderlich ist, um die innere Arbeit der Umlagerung der Struktur zu leisten, konnte für die Stoff wechselprodukte der Gewebsparasiten bisher nicht nachgewiesen werden. Nach unseren, noch nicht abgeschlossenen Untersuchungen über die Teerwirkung, wird es vielleicht gelingen, diese Forderung zu erfüllen, trotzdem, wie später berichtet werden wird, hier zur Zeit noch technischeSchwierigkeiten bestehen. Das Problem müßte dann, wie oben erwähnt, auf einem anderen Gebiet in Angriff genommen werden: durch experimentelle Erzeugung von Röntgencarcinomen, wo die auslösende Energie sich qualitativ und quantitativ voraussichtlich mit aller wünschenswerten Präzision feststellen lassen wird. Es sind nun auch als auslösende Faktoren des Teers resp. der Steinkohlendestillationsprodukte verschiedene organische und anorganische Bestandteile des Materials angenommen worden. Auf die verschiedenen, 'aus der Anilinfarbenindustrie verantwortlich gemachten organischen Körper kann hier mangels eigener Erfahrungen nicht eingegangen werden, eine kurze Besprechung verlangen aber die Mitteilungen der Belgier Slosse und Bayet ${ }^{36}$ ), daß der wirksame Faktor bei den von ihnen untersuchten schweren Industrieschädigungen im Kohlengewerbe, durch einen regelmäßig, nach ihren Mitteilungen sehr erheblichen Gehalt der Steinkohlenprodukte an Arsen bedingt ist. Sie fanden in dem Ausgangsmaterial der belgischen Steinkohle bis zu $0,06 \%$ Arsen.

Die Gefahr, eine chronische Arsenvergiftung zu bekommen, geht aus ihren Befunden noch daraus hervor, daß bei allen Arbeitern, die dem Teerstaub ausgesetzt sind, Arsen in den Haaren, bei den meisten auch im Blut und Harn gefunden wird, ebenso ließ sich Arsen in der Luft des Arbeitsraumes der erkrankten Arbeiter nachweisen. Positive Arsenbefunde wurden von ihnen außerdem im Ofenruß (Schornsteinfegerkrebs!), in den Behältern, in denen Leuchtgas gereinigt wurde, in Schmierölen und in fünf von ihnen untersuchten Anilinfarben (Ba yerLeverkusen) erhalten. Der von Fibiger angewandte Steinkohlenteer enthielt ebenfalls Arsen, wenn auch nur $0,0003 \%{ }^{7},{ }^{8}$ ). 
Bei Bestätigung dieser Behauptung würde sich demnach die schädigende Wirkung der Steinkohle und ihrer Produkte auf einen, ihnen allen gemeinsamen Nenner - das Arsen - zurückführen lassen.

Das würde nicht nur die ganze Prophylaxe in der Industrie erheblich vereinfachen lassen, sondern auch eine Erkenntnis für die Pathogenese maligner Neubildungen ergeben, die bei experimenteller Einstellung des Arsens als spezifisch blastogenen Faktors, die von mir bereits in einer früheren Arbeit ${ }^{2}$ ) postulierten Eigenschaften des auslösenden Faktors in denkbar günstiger Form ergäbe.

Nach unseren Untersuchungen muß aber diese Behauptung jedenfalls in ihrer generellen Fassung aus folgenden zwei Gründen abgelehnt werden.

Nach der Analyse des Steinkohlenteers, für deren Ausführung ich Herrn Prof. Göhlich vom hiesigen chemischen Staatslaboratorium zu großem Dank verpflichtet bin, enthält unser Präparat (Mitteilung vom 9. IV. 21) $0,18 \%$ Mineralbestandteile mit $0,05 \%$ Eisen, während Arsen sich in ihm nicht nachweisen läßt. Wir haben also unsere Hautcarcinome mit einem arsenfreien Teer erzeugt. - Da angenommen werden konnte, daß die Teerwirkung evtl. durch Arsenbeimengungen gesteigert wird, erhielten zwölf Tiere täglich - 4l Tage lang - zuerst Arsen allein $(0,001$ Sol. Kalii arsenicossi $=0,00001$ As. $)$, dann weiter Arsen und gleichzeitig Teer. Das Resultat dieser kombinierten Behandlung ist insofern bemerkenswert, als bisher keines der so behandeiten Tiere irgendeine der sonst regelmäßigen Teerschädigungen der Haut gezeigt hat. Die zum Teil früh (71. bis 96. Tag der Gesamtbehandlung) gestorbenen Mäuse kommen für die Bewertung dieser Behandlung nicht direkt in Betracht, die den für das Auftreten der Papillome kritischen 60. Tag überlebenden Tiere, speziell eine Maus, die zurzeit 120 Tage in Behandlung ist, zeigten bisher - abgesehen von der typischen Enthaarung - keine Hautveränderung.

Die Untersuchungen unserer mit Teerpinselung behandelten Mäuse, die von cand. med. E. Möller - ausgehend von anderen Gesichtspunkten - ausgeführt sind und demnächst ausführlich mitgeteilt werden sollen, gingen darauf aus, ein möglichst vollständiges Bild von den gesetzten Schädigungen zu erhalten.

Neben der Beobachtung der lokalen Schädigungen der Haut wurden deshalb in regelmäßigen Abständen die allgemeinen Schädigungen des Organismus durch Harnanalysen, Kontrolle des Körpergewichts, des motorischen Verhaltens und durch hämatologische Untersuchungen verfolgt. Die lokalen Hautveränderungen wurden an exzidierten Stücken der geteerten Haut periodisch kontrolliert. Vom ersten Behandlungstage $a b$ suchten die Mäuse den eingepinselten Teer mit Zähnen und Extremitäten zu entfernen; es ist anzunehmen, daß hierbei nicht unbe- 
trächtliche Teermengen verschluckt wurden. Veränderungen am Verdauungsapparat, die hierauf zurückführbar wären, wurden von uns jedoch nicht beobachtet, die perorale Zufuhr erklärt aber vielleicht besser das frühe Auftreten der Nierenveränderungen.

Nach etwa 2 Wochen fallen im Bereich der Teerapplikation die Haare auch spontan aus, so daß die Epidermis völlig depiliert und glatt vorliegt. Bei der mikroskopischen Untersuchung der Haut lassen sich jedoch im Corium auch noch in späteren Stadien Rudimente des Haares resp. die Haarwurzeln nachweisen.

Die erste nachweisbare Allgemeinschädigung ist durchweg eine Albuminurie, die bei einigen daraufhin untersuchten Tieren am ersten Behandlungstag festgestellt wurde, bei den übrigen am 3 . bis 8 . Behandlungstag nachgewiesen ist und bis zum Exitus bestehen blieb. Im Sediment des Harns fanden sich rote Blutkörperchen und spärliche hyaline Zylinder in geringen Mengen.

Im Laufe des ersten und zweiten Behandlungsmonats nahm das Körpergewicht bei etwa $40 \%$ der Tiere für die Dauer einiger Wochen zu, um danach langsam bis zum Exitus abzusinken. Da sich bei der mikroskopischen Untersuchung von Hautstücken aus dieser Periode ein bis auf die Muskelschicht der cutis reichendes Ödem nachweisen ließ, scheint diese Gewichtszunahme auf allgemeine Ödeme zurückführbar zu sein. Im Laufe des ersten und zweiten Monats wird das Fell struppig, und bei einzelnen Tieren tritt für die Dauer mehrerer Wochen eine große Schreckhaftigkeit und motorische Unruhe auf. All diese Symptome wurden von uns auf die bei der mikroskopischen Untersuchung nachgewiesenen nephrotischen Schädigungen bezogen.

Hämatologisch ließ sich in den ersten Behandlungswochen ein Anstieg der absoluten Leuko- und Lymphocytenwerte feststellen. An der Haut trat zuerst eine Auflockerung und Verdickung, etwa von der zweiten bis dritten Woche $a b$ eine auffallende Trockenheit und Rissigkeit auf. Die ersten Hyperkeratosen wurden nach etwa 3 Wochen, die ersten Papillome nach ca. 60 Tagen, die ersten breitbasigen, in die Tiefe gehenden Tumoren, die makroskopisch als Carcinombildung imponierten, etwa am 110. Tage der Behandlung notiert.

Von den Tieren starben im Laufe des ersten und zweiten Monats $0 \%$, im dritten $24 \%$, vierten $12 \%$, fünften $15 \%$, sechsten $24 \%$ und siebenten und achten $25 \%$.

Die mikroskopische Untersuchung wurde an Paraffinschnitten nach Fixierung in Carnoy, danach Alkohol, Xylol vorgenommen. Gefärbt wurde anfangs nur mit Hämatoxylin-Eosin, später wurden alle Präparate außerdem mit MethylgrünPyronin, nach Unna Pappenheim, May-Grünwald, Giemsa, zum Teil noch mit Resorcin-Fuchsin und nach van Gieson gefärbt. Diese verschiedenen Färbungen wurden vorgenommen, weil wir im subepithelialen Gewebe Zellen fanden, deren Provenienz und Funktion wir aus ihrem färberischen Verhalten festzustellen suchten. 
Zur Charakterisierung der epithelialen Bildungen muß angeführt werden, da $B$ diese im allgemeinen im Laufe der ersten 60 Tage sich in einer mehr oder weniger starken Epithelhyperplasie, etwa vom 60. Tage ab in der Bildung stark verzweigter Papillome äußert, und daß erst etwa vom 110. Tage ab das distale infiltrative Tiefenwachstum nachweisbar wird. Maligne Bildungen in den einmal entstandenen Papillomen wurden nur einmal beobachtet. Beim Tiefenwachstum dringen unregelmäßig über den geschädigten Bezirk der Haut verteilte Zapfen des Stratum basilare in die bis dahin mäßig stark mit Leukocyten und den gleich zu besprechenden Zellen infiltrierte subepitheliale Schicht ein. An dem distalen Ausläufer dieser Zapfen sieht man häufig die großen Zellen mit ihrem blasigen Kern aufgelagerte Kappen aus Zellen mit dunkler sich färbenden Kernen. Die Zahl der Mitosen, besonders die Stadien bis zur Bildung der Äquatorialplatte, sind besonders reichlich vertreten. Den Teer findet man zum Teil in zusammenhängenden, gelbbraunen, mehr homogenen Massen dem Epithel aufgelagert, sehr selten zwischen den Epithelzellen, und dann wieder in der subepithelialen Schicht, die hier in ihren obersten Partien zahlreiche Histiocyten zeigt, die in den Maschen des Bindegewebes liegen und meist eine, seltener zwei oder mehrere große, unregelmäßig geformte Zellen umschließen, die bei Hämatoxylin-Eosinfärbung durch die helle, wasserblaue Farbe ihrer feindispersen Protoplasmastruktur und den großen blasigen, ein lockeres Randchromatinnetz zeigenden Kern ausgezeichnet sind. Außer diesen Zellen fanden wir keine Plasma- und nur spärliche Mastzellen. Eosinophile Zellen wurden von uns nicht beobachtet. Die zahlreichen Blutcapillaren sind häufig mit Leukocytenthromben verstopft, zeigen nicht selten eine Verbreiterung der Endothelien, keine auffallenden Wandveränderungen. Capillare Blutungen sind reichlich über das ganze Gewebe bis an die Muskelschicht verteilt. Während die meisten der Zelltypen im Bindegewebe sich also leicht bestimmen lassen, haben wir die Provenienz und Funktion der zwei immer im engsten Kontakt miteinander auftretenden Zelltypen nicht mit Sicherheit feststellen können, weil sie nach ihrem färberischen Verhalten sich keiner der feststehenden Arten völlig einfügen. Und zwar handelt es sich 1. um den Nachweis der Provenienz der Granula in den Histiocyten, 2. um die Art der großen, von ihnen umlagerten Zellen. Es sind mikroskopisch 2 verschiedene Pigmentgranula zu beobachten: gelbbraune, glänzende, relativ gleichgroße, rundliche und schwarzbraune ungleichgroße. Die gelbbraunen geben eine ausgesprochene Eisenreaktion (Turnbull und Berlinerblau), doch bleiben neben den blau sich färbenden Granulis noch zahlreiche feiner verteilte ungefärbt. Es ist nicht daran zu zweifeln, daß diese Granula demnach Eisen enthalten und aus den zahlreichen extravasierten Erythrocyten abgebautes Hämosiderin sind; daß ein Teil von ihnen, und vor allem die unregelmäßigen, schwarzbraunen Pigmentgranula-Teerpartikel sind, die das Eisen, das der Teer zufolge der chemischen Analyse enthält, hier an ihre Teilchen adsorbiert enthalten, scheint ebenso mit Sicherheit anzunehmen zu sein. Dafür, daß die gelbbraunen, relativ gleichmäßig geformten Granula als Hämosiderin aufzufassen sind, sprechen die gleichzeitigen frischen capillaren Blutungen, nach denen Bedingungen zum Blutaustritt im Gewebe vorliegen müssen. Die Gründe, die für die Provenienz der 2. Granulaart aus dem Teer sprechen, sind in ihrer auffallenden Häufung in der Grenzschicht zum Stratum basilare gegeben, wo die engen Bluteapillaren des Stratum papillare besonders zu Diapedese und Rhexis neigen, wo aber auch die größte Häufung des einwandernden Teers zu erwarten ist, zweitens in der nur partiellen Eisenreaktion der gelben Granula, die aber ebenso bei einwandfreiem Hämosiderin zu beobachten ist, drittens darin, da $\beta$ sich diese Granula schon in frühesten Stadien der Teerpinselung (20. Tage) finden, wo das Bindegewebe frei von Blutaustritten erscheint. Alle Präparate sind nach der Fixierung in Alkohol 
entwässert und in Xylol aufgehellt, und erst nachträglich kam uns die Überlegung, daß durch diesen Proze $B$ die größte und voraussichtlich die feinstverteilte Menge des Teers gelöst und entfernt worden sein kann. Um diese möglicherweise artefizielle Ursache der relativen Teerarmut an dem fixierten Material festzustellen, wurden die Schnitte mit Chloroform, Xylol oder Alkohol nachträglich nochmals behandelt, und hierbei ging nachweislich ein größerer Prozentsatz der Granula in Lösung, was wiederum für ihren Teercharakter sprechen würde.

Die 2. Frage betrifft die zentral gelagerten großen Zellen mit hellem Protoplasma und großem, blasigem Kern. Diese zeigen bei Methylgrün-Pyronin rote, grobe Granula, mit Panchrom (Unna) feinere blaue, mit Giemsa blauviolette und mit May-Grünwald blaue Granula. Der Zell- und Kernform nach können es große Wanderzellen sein [Herzog${ }^{14}$ ), Marchand $\left.\left.{ }^{22},{ }^{23}\right)\right]$, die von den Deckepithelien der Gefäße abgewandert und im Úbergangsstadium teils zu großen lymphoiden Zellen, teils zu großen Granulocyten sind. Diese Auffassung würde ihr atypisches Verhalten gegenüber den erwähnten Farbstoffen erklären; warum diese Zellen aber fast regelmäßig im Zentrum der mit dem Pigmenttransport beschäftigten Histiocyten liegen, ob sie mit diesen zusammenhängen, oder ob sie evtl. spezifische Reaktionsprodukte auf den Krebs auslösenden Teer sind, können wir nicht angeben. Sobald sie zu zweien oder mehreren zusammenliegen, hat die Zahl der sie umlagernden pigmenthaltigen Histiocyten abgenommen.

Beim Vordringen des Carcinoms läßt sich ein Zusammenhang seiner Zellen mit den eben beschriebenen Histiocyten und mit den Wanderzellen nicht erkennen. Das Krebsgewebe besteht aus ziemlich gleichgroßen, unregelmäßig polygonalen Zellen mit großem, blasigem Kern, die an einzelnen Stellen nach Form und Anordnung den Eindruck von Sarkomgewebe machen. Reine Sarkombildung wurde von uns, im Gegensatze zu dem einen Fall Tsutsuis, nicht beobachtet. Über Metastasenbildung und Transplantierbarkeit, die nicht in direktem Zusammenhang mit der hier untersuchten Frage stehen, wird an anderer Stelle berichtet.

Unter Benutzung der physikalisch-chemischen Vorstellungen, die wir über die schädigende Wirkung eines Destillationsproduktes des Teers - der Phenole - haben, läßt sich dem Problem der Teerwirkung in der Haut physikalisch-chemisch nähertreten.

Im Blut der Niere zugeführte Stoffe treten in die Nierenzellen ein und werden, da sie in den Blutkolloiden gelöst sein müssen, durch Osmose mit der Struktur des Nierenzellprotoplasmas in Reaktion treten. Sie werden hier wahrscheinlich an der Structuroberfläche, den Granulis, die an bestimmten Epithelien als spezifisch reagierend vorzustellen sind, adsorbiert und führen dann zu Strukturumlagerungen des kolloidalen Systems, die sich, je nach der Zellindividualität, in Zunahme oder Abnahme der dispersen Phase äußern (Gel - Solzustand). Daß mit dem Zustand der dispersen Phase des Zellinnern prinzipielle Änderung der Systemfunktionen gegeben sind, wissen wir nicht nur aus der bei der Phenolwirkung spezifisch auftretenden Nephrose, sondern kennen diesen Zustand vor allem in seiner prinzipiellen Bedeutung für die Aktivität jedes dispersen Systems. Ein Úbergang einer feindispersen Phase in eine grobdisperse, resp. der umgekehrte Vorgang bedingt zwangsläufig im ersten Fall eine Abnahme, im umgekehrten eine Zunahme der energetischen Funktionen. Bei der Nierenwirkung ist besonders eine 
Phase dieser Funktion studiert worden - die diuretische Wirkung der eiweißfällenden Salze [Hof meister $\left.\left.{ }^{15}\right)\right]$.

Wenn wir, wie im Fall der Teerwirkung auf das Hautepithel, die Teerpartikel am Reaktionsort bisher nur mikroskopisch, $d . h$. in relativ grober Verteilung nachweisen konnten, so läßt sich hieraus kein weiterer Schlu $\beta$ ziehen, als daß in ihnen das Gesamtdepot der wirksamen Substanz gegeben ist, während der Nachweis ihrer voraussichtlich erst spezifisch wirksamen feindispersen Aufteilung uns bisher nicht gelang. Deswegen wurden auch die verschiedenen Granulafärbungen versucht, um aus ihnen, wenn möglich, einen morphologisch nachweisbaren, wenn auch indirekten Anhaltspunkt für die Wirkung des Teers zu gewinnen. Allem Anschein nach ist eine Ableitung aus den beschriebenen, sich färberisch unspezifisch verhaltenden Granulationen der Wanderzellen nicht zulässig, sondern diese sind als Reaktionsprodukte auf die hämosiderinhaltigen Zellen aufzufassen.

Wir können nach dem momentanen Stande unserer Untersuchungen also nur das wirksame Material, wenn auch in noch unwirksamer Phase, nachweisen und sind für die Erklärung seiner auslösenden Wirkung fürs erste auf allgemeine Ủberlegungen angewiesen.

\section{Das Wesen des ,Reizfaktors" und die wechselseitige Reaktion von Reizfaktor und Gewebe.}

Bei mikroskopischer Beobachtung einfacher Protoplasmastrukturen, z. B. von Leukocyten, sieht man regelmäßig bei Durchleitung eines elektrischen Stroms, daß ihre Bewegung aufhört und sie sich unter Verdichtung der Struktur zusammenziehen. Es ist dieses ein einfaches Beispiel einer Reaktion auf einen von außen zugeführten Reiz, der an der Oberfläche der Struktur angreift und zu Veränderungen der inneren Struktur (reversibler Übergang in eine gröber disperse Phase) führt. - Wenn die biologischen Vorgänge, die wir am befruchteten Seeigelei - wie die Bildung der Befruchtungsmembran, die Furchungseinleitung - auf die Wirkung des eingedrungenen Spermatozoens zurückführen, durch $\mathrm{Zu}$ fuhr bestimmter chemischer Körper willkürlich hervorzurufen sind, so illustriert diese Methode ebenfalls die Reaktion der Struktur, auf einen von außen zugeführten Reiz, der diesmal ein chemischer ist. Diese wirksamen Reizfaktoren lassen sich, nu ihrem Wesen nach, als energetische Faktoren charakterisieren, die infolge ihrer Konstitution imstande sind, an der Struktur des Objekts bestimmte Veränderungen hervorzurufen.

Der ganze biologische Prozeß läßt sich demnach vereinfacht vorstellen als Reaktion zwischen dem kolloidalen energetischen System der betreffenden Zellen und einem von außen zugeführten zweiten energetischen System. Hierdurch gewinnen wir, an Stelle der verschwomme- 
nen, teleologischen Begriffe des formativen, entzündlichen, und wie für unsere Untersuchungen gezeigt wird, blastogenen Reizes einerseits und an Stelle des unbestimmten Begriffes der Zelle andererseits, gut definierbare Vorstellungen.

Aus der kritischen Wertung der von uns angewandten experimentellen Methoden hat sich ergeben, daß nur diejenigen praktischen Erfolg haben, die den auslösenden Faktor dem Erfolgsorgan in Form solch einer freien Energie zuführen, also den theoretischen Forderungen entsprechen. Diese Eigenschaften sind gegeben sowohl in den Stoffwechselprodukten bestimmter Gewebs- oder Zellparasiten, vom Typus der Nematoden, Protozoen, Chlamydozoen - bestimmter isolierter chemischer Körper vom Typus des Teers, von physikalischen Einwirkungen vom Typus der Röntgen- und der Radiumstrahlen, also - allgemein gefaßt - in der Wirkung nichtmechanischer Energiearten, die eine molekulare Umlagerung der Struktur in dem betreffenden Energiesystem der Zelle hervorrufen. - Die Reaktion erfolgt je nach den wechselseitigen Intensitäten (Affinitäten) und Kapazitäten (Stoffmengen), d. h. nach der Konstitution der energetischen Systeme, und hierbei wird das relativ metastabile, makroheterogene System des Objekts in ein labiles, mikroheterogenes System umgelagert. Das auslösende Energiesystem greift dabei mittelbar an der Struktur an, wird also voraussichtlich nach den allgemeinen physikalisch-chemischen Gesetzen der Molekularchemie wirken. Solange es chemische Körper sind, wird es auf dem Lymph-oder Blutwege zugeführt und im Gewebe am Erfolgsorgan deponiert, die strahlende Energie, die allem Anschein nach indirekt ebenfalls als chemische Energie wirkt ${ }^{16}$ ), würde demnach unter prinzipiell gleichen Bedingungen angreifen.

Daß in der Tat solch eine Erhöhung der dispersen Phase der Kolloide auch am energetischen System der Zellen im Tumor vorliegt, wurde von Kottmann ${ }^{20}$ ) nachgewiesen. Durch den neugeschaffenen hochdispersen Zustand seiner Kolloide erhält das ganze System eine ungeheure Steigerung seiner physikalischen und chemischen Reaktionsfähigkeiten. Formal ist diese ausgedrückt in dem hellen, feindispersen Zellprotoplasma, in dem hellen, blasigen Kern mit seinem Reichtum an Mitosen, biologisch in dem erwähnten, von Kottmann nachgewiesenen verschiedenen Verteilungsgrad der Blutkolloide im Kapillargebiet der carcinomatösen und der nicht carcinomatösen Organe. Zeigt ein Normalserum nach Reaktion mit Normalorganen den Verteilungsgrad I, so beträgt dieser mit Carcinomgewebe 24. Da nach seinen Untersuchungen dieser hohe Dispersitätsgrad der Blutkolloide regionär auf das Tumorgewebe beschränkt ist, die anderen Gewebe des Körpers aber nur niedrige Dispersion der ihnen zugeführten Blutkolloide erzielen können, reißt der Tumor alle wertvollen Nährsubstanzen an sich und garantiert 
damit so schon indirekt sein erhöhtes Wachstum, während die anderen Organe, denen er die Nahrung wegfängt, kachektisch zugrunde gehen. Der damit erreichte pathologische Verjüngungszustand des Tumorgewebes zeigt sich in gesteigertem Wachstum, gesteigerter Vermehrungsfähigkeit, zugleich in dem relativen Verlust der spezifischen Organfunktionen. Daß3 diese Verteilungsbedingungen der Blutkolloide auch für die zerstörende Einwirkung auf andere Strukturen verantwortlich gemacht werden können, erscheint unwahrscheinlich. In der Konstitutionsänderung des pathologischen Energiesystems sind aber noch andere Faktoren gegeben, die zur Erklärung weiterer charakteristischer Eigenschaften des Tumors heranzuziehen sind. Vor allem sind diese aus der Untersuchung des Ladungssinnes der Teilchen (Moleküle, Ionen) zu erwarten. Unsere eigenen Untersuchungen haben bei Kataphoreseversuchen bisher zu einem negativen Resultat geführt. - Ein weiterer, dabei indirekter Nachweis der Konstitutionsänderung ist schließlich aus der Reaktion der neugebildeten Strukturen auf eine von außen zugeführte dosierte Energie zu erwarten. Hierüber liegen auf dem Gebiet der experimentellen Strahlenforschung eine Reihe wertvoller Beobachtungen vor.

Nach dem Gesetz von Bergonié und Tribondeau ${ }^{16}$ ) wirken Röntgenstrahlen um so intensiver schädigend auf die Zelle, ,je größer ihre reproduzierende Fähigkeit, je länger ihr karyokinetischer Werdegang und je weniger ihre Morphologie und ihre Funktionen endgültig festgelegt sind". Dieses aus dem Verhalten der spezifischen Hodenzellen abgeleitete Gesetz würde, bezogen auf die gleichen für die Tumorzelle typischen Eigenschaften, diese in dem eben besprochenen Sinne als äußerst strahlenempfindliche Struktur charakterisieren. Der Effekt, der bei Röntgenbestrahlung angestrebt wird, besteht in der letalen Schädigung der Tumorzellen. An Präparaten von röntgenbestrahltem Carcinomgewebe läßt sich nun nachweisen [Reichold $\left.{ }^{31}\right)$ ], daß dieser Effekt damit erreicht wird, daß der hochdisperse Verteilungszustand der kolloiden Systeme in den pathologischen Zellen in einem grobdispersen übergeführt wird, bei dem ihre Lebensfunktionen aufhören.

Theoretisch ist es denkbar, daß dabei den pathologischen Strukturen genau so viel Energie zugeführt wird, daß das Plus ihrer Energie-. steigerung durch die entgegengesetzt gerichtete, energieherabsetzende Röntgendosis aufgehoben und die ,Ausgangspunkte" neuer hochdisperser Phasen zugleich zerstört werden.

Bekanntlich wird ein solch ideales Resultat nur selten und unter besonders günstigen bestimmten Bedingungen erreicht. Dieser Befund an sich charakterisiert aber die Zellstrukturen der malignen Tumoren als energetische Systeme, deren Bildung und Funktionen nach physikalisch-chemischen Gesetzen erfolgen. 


\section{Literaturverzeichnis.}

1) Belogolow y, G., Arch. f. Entwicklungsmech. d. Org. 43, 556/693. 1918. $\left.{ }^{2}\right)$ Bierich, R., Zeitschr. f. Krebsforsch. 18, 71. 1921. - ${ }^{3}$ ) Borst, M., Ergebn. d. allg. Pathol. u. pathol. Anat. 4. 1897. $-3^{a}$ ) Zieglers Beiträge z. allg. Pathol. u. pathol. Anat. 49, 638. 1910. - ${ }^{4}$ ) Borst, M., Aschoffs Lehrb. d. pathol. Anat. 1919. - ${ }^{5}$ ) Eichwald, E., Die phys.-chem. Grundlagen der Biologie, 1919, S. 338, 391, 456. $-{ }^{6}$ ) Fibiger, I., Zeitschr. f. Krebsforsch. 17. 1919. - ${ }^{7}$ ) Fibiger, I., und Fr. Bang, Cpt. rend. des séances de la soc. de biol. 83, 20. 1920. - Fibiger, I., und Fr. Bang, Experiment. product. of tar cancer in white mice. Kopenhagen 1921. - $\left.{ }^{9}\right)$ Fischer, E., Ber. d. Dtsch. chem. Ges. 39, 530. 1906. - ${ }^{10}$ ) Hart mann, A., Arch. f. mikr. Anat. 76, 252. 1910. - 11) Heidenhain, H., Plasma und Zelle. 1911. - ${ }^{12}$ ) Held, Die Entwicklung des Nervengewebes bei Wirbeltieren. 1909. - 13) Held, Über die Jeuroglia marginalis der menschlichen Großhirnrinde. Monatsheft f. Psychol. u. Neurol. 16. 1909. - $\left.{ }^{14}\right)$ Herzog, G., Zieglers Beiträge z. allg. Pathol. u. pathol. Anat. 61, 325/450. 1916. - 15) Hof meister, Arch. f. experim. Pathol. u. Pharmakol. 24. 1888. - ${ }^{16}$ ) Holth usen, H., Pflüger's Arch. f. d. ges. Physiol. 18\%. 1920, mit Zitat Bergonié und Tribondeau. 17) Hueck, W., Zieglers Beiträge z. allg. Pathol. u. pathol. Anat. 66. 1920. 18) Jamagiwa und Ischikawa, Mitt. d. med. Ges. zu Tokio 15. 1915; 17. 1917; 19. 1919. - ${ }^{19}$ ) v. Korff, Arch. f. mikr. Anat. 84, 263/264. 1914. - 20) Kottmann, K., Schweiz. med. Wochenschr. 50, 47. 1920. - ${ }^{21}$ ) Lubarsch, O., 16. internat. Kongreß d. Med. Budapest, Skt. 3. 1909. - ${ }^{21}$ a) Lubarseh, O., Med. Klin. 41, 1651. 1912. - ${ }^{22}$ ) Marchand, F., Verhandl. d. dtsch. pathol. Ges. 5. 1913. - ${ }^{23}$ ) Marchand, F., Handb. d. allg. Pathol. 1. 1908. - ${ }^{24}$ ) Mewes, F., Arch. f. mikr. Anat. 75. 1910. - ${ }^{25}$ ) Me yer, R., Zeitschr. f. Geburtsh. u. Gynäkol. 71. 1912. - ${ }^{26}$ ) Mollier, Arch. f. mikr. Anat. r4. 1909. - ${ }^{27}$ ) Murphy, I., Journ. of experim. med. 3, 4. 1921. - 28) Ostwald, Wo., Grundriß der Kolloidchemie. 1912. $-{ }^{29}$ ) Orth, I., Zeitschr. f. Krebsforsch. 10. 1911. 30) Ra ncke, O., Heidelb. Akad. Mathem. Naturw. Klasse B 3. 1913; 2. 1914. $\left.{ }^{31}\right)$ Reichold, Münch. med. Wochenschr. 28. 1921. - ${ }^{32}$ ) Ribbert, Beiträge zur Entstehung der Geschwülste. Bonn 1906. - ${ }^{33}$ ) Rohde, E., Vortr. u. Aufs. üb. Entwicklungsmech. d. Org. 20. 1914. - ${ }^{34}$ ) Rückert, Hertwigs Handb. d. vergleich. experim. Entwicklungslehre 1. 1906. - ${ }^{35}$ ) Schaper und Cohen, Arch. f. Entwicklungsmech. d. Org. 19. 1905. - ${ }^{36}$ ) Slosse und Bayet, Ann. et bullet de la soc. roy. des sciences med. et natur. de Bruxelles 1920, Nr. 2, 3. ${ }^{37}$ ) Studnicka, Anat. Anz. 40. 1912. - ${ }^{38}$ ) v. Szilly, Anat. Hefte 35. 1908. 39) The Svedberg, Kolloidchem. Beihefte 5. 1921. - 40) Teutschländer, O., Verhandl. d. dtsch. pathol. Ges. 31. 1921. - $\left.{ }^{41}\right)$ Tsutsui, H., Gann. 12. 1916. Zitiert nach Fibiger. - ${ }^{42}$ ) Versé, M., Entstehung, Wachstum und Bau der Polypen, Adenome und Carcinome. Leipzig 1908. - ${ }^{43}$ ) Weimarn, P. v., Grundzüge der Dispersoidchemie. Dresden 1911. - ${ }^{44}$ ) Wetzel, E., Münch. med. Wochenschr. 29, 910.1921. 